\title{
DIVERSITY PATTERNS AND CONSERVATION STATUS OF NATIVE ARGENTINEAN CRUCIFERS (BRASSICACEAE)
}

\author{
Diego L. Salariato (iD \& Fernando O. Zuloaga
}

Instituto de Botánica Darwinion (CONICET-ANCEFN), Labardén 200, Casilla de Correo 22, B1642HYD San Isidro, Buenos Aires,Argentina; dsalariato@darwin.edu.ar (author for correspondence).

\begin{abstract}
Salariato, D. L. \& F. O. Zuloaga. 2020. Diversity patterns and conservation status of native argentinean crucifers (Brassicaceae). Darwiniana, nueva serie 8(2): 530-566.

Global warming, coupled with habitat destruction and human activity, are accelerating the rates of species extinction worldwide. Species-extinction risk assessment using the IUCN Red List categories, together with the study of the spatial patterns of biodiversity, are fundamental approaches for identifying conservation priorities and targeting government decisions to mitigate impacts on biodiversity. Here, we analyzed the geographic distribution of Argentinean species of Brassicaceae using species point distributional data. In this way, we classified species following the IUCN threat categories at a regional level, and analyzed patterns of richness, endemism, and threat on the different ecoregions and biomes of the country. In addition, we also explored differences in elevation, annual mean temperature, annual precipitation, and aridity between endemic vs. non-endemic and threatened vs. non-threatened species. The results showed that of the 162 Argentinean taxa, 58 species were here categorized as threatened (VU, $\mathrm{EN}$, or CR) (36\%). However, when only endemics are considered, more than half of these (33 spp, $57 \%$ ) are threatened. Although species inhabit all environments and biogeographic regions of the country, arid to semi-arid areas, which are associated to the Andes and the Patagonian steppe, contained most of the species. Specifically, the Central Andean Puna and the Patagonian steppe ecoregions included the greatest number of species, endemics, and threatened species. Furthermore, different hotspots of richness, endemism, and threat were detected along Andean regions and the Patagonian steppe, and endemics were characterized by inhabiting on average drier areas than non-endemic native species. An up-to-date species list, including conservation status, distribution maps for all species, hotspots of richness, endemism, and threat, are also provided. This work seeks to contribute to the knowledge on geographical patterns of the Argentinean flora and its conservation, complementing the information published in the Flora of Argentina.
\end{abstract}

Keywords. Andes; Argentina; Central Andean Puna; conservation; endemics; IUCN; Patagonian steppe; richness.

Resumen. Salariato, D. L. \& F. O. Zuloaga. 2020. Patrones de diversidad y estado de conservación de las crucíferas argentinas nativas (Brassicaceae). Darwiniana, nueva serie 8(2): 530-566.

El calentamiento global junto con la destrucción de hábitats y la actividad humana están acelerando las tasas de extinción de especies en todo el mundo. La evaluación del riesgo de extinción de especies utilizando las categorías de la Lista Roja de la UICN, junto con el estudio de los patrones espaciales de la diversidad biológica, son enfoques fundamentales para determinar las prioridades de conservación y orientar las decisiones gubernamentales para mitigar los impactos en la diversidad biológica. En este trabajo analizamos la distribución geográfica de las especies argentinas pertenecientes a la familia Brassicaceae utilizando datos precisos sobre la distribución de las especies. De esta forma, determinamos su clasificación en las categorías de amenaza de la UICN a nivel regional, y analizamos los patrones de riqueza, endemismo y amenaza en las diferentes ecorregiones y biomas del país. 


\begin{abstract}
Además, exploramos las diferencias en elevación, temperatura media anual, precipitación anual y aridez entre especies endémicas vs. no endémicas y amenazadas vs. no amenazadas. Los resultados muestran que de los 162 taxones argentinos, 58 especies fueron categorizadas aquí como amenazadas (VU, EN, o CR) (36\%). Sin embargo, cuando se consideran sólo los endemismos, más de la mitad de las especies endémicas (33 spp, 57\%) están amenazadas. Aunque las especies habitan en todos los ambientes y regiones biogeográficas del país, las zonas áridas y semiáridas asociadas a los Andes y la Estepa Patagónica contuvieron la mayoría de las especies. En particular, las ecorregiones de la Puna Central Andina y la Estepa Patagónica incluyeron el mayor número de especies, especies endémicas y especies amenazadas. A lo largo de las regiones andinas y de la estepa patagónica se detectaron diferentes zonas de elevada riqueza, endemismo y amenaza; las especies endémicas se caracterizaron por habitar zonas en promedio más secas que las especies nativas no endémicas. También se proporciona una lista actualizada de las especies con el estado de conservación, mapas de distribución de todas las especies y puntos de elevada riqueza, endemismo y amenaza. Este trabajo tiene por objeto contribuir al conocimiento de los patrones geográficos de la flora Argentina y a su conservación, complementando la información publicada en la Flora de la Argentina.
\end{abstract}

Palabras claves. Andes; Argentina; endemismos; Estepa patagónica; conservación; Puna Andina Central; riqueza; UICN.

\section{INTRODUCTION}

The current global extinction crisis presents a great ecological challenge, and the loss of biodiversity is one of the most critical ongoing environmental problems (Dirzo \& Raven, 2003; Ceballos et al., 2015). Global warming, coupled with habitat destruction and degradation, are causing substantial species-range shifts, contractions and local extirpations, which can be major causes of biodiversity loss (Brook et al., 2008; Chen et al., 2011; Urban, 2015). However, the need for conservation actions sometimes contrasts with the considerable uncertainty about the status of threats (Corlett, 2016; Bachman et al., 2018) and the spatial distribution of diversity (Whittaker et al., 2005; Bini et al., 2006). Species extinction risk assessment following the IUCN Red List categories and criteria (International Union for Conservation of Nature, www.iucn. org) is widely recognized as one of the most useful approaches for identifying conservation priorities and targeting government decisions to mitigate impacts on biodiversity (Ricketts et al., 2005; Rodriguez et al., 2006; Brooks et al., 2015; Bennun et al., 2018; Nic Lughadha et al., 2020). Although IUCN Red List Category data are produced on a global scale, is also essential to establish IUCN threat categories at the regional level in order to implement concrete conservation actions (Gärdenfors et al., 2001).
Argentina includes a high number of ecoregions due to its latitudinal and altitudinal diversity (Lean et al., 1990; Morello et al., 2018). Likewise, the Argentinean Flora, distributed along 13 biogeographical provinces sensu Cabrera \& Willink (1980) or 17 ecoregions and six biomes sensu Olson et al. (2001), presents the greatest number of species in the Southern Cone region, comprising ca. 9237 species, of which $1731(\sim 18 \%)$ are endemic to the country (Zuloaga et al., 2019). Within it, the mustard family (Brassicaceae) is well represented by eleven tribes, 35 genera, 160 species, and two subspecies that are distributed mainly along the Andes, but with representatives in all biogeographic provinces and biomes. During the last 50 years, taxonomic revisions and systematic studies, several regional floras and checklists have focused on the distribution of the family in the country (Boelcke 1967; 1987; Boelcke \& Romanczuk 1984a; 1984b; Boelcke \& Martínez-Laborde, 1994; Prina, 1995a; 1995b; Martínez-Laborde, 1999; Al-Shehbaz, 2008), but it was not until the publication of the flora of Argentina (Al-Shehbaz, 2012a) that the family had its most comprehensive treatment. Nevertheless, the study of the species geographic ranges, their threat categorization, the association with the different ecoregion/ biomes, and an integrative analysis of its distribution in the country are still pending.

Argentinean crucifers seem to be mainly distributed along the central and southern Andean highlands $\left(\sim 22^{\circ}\right.$ to $55^{\circ} \mathrm{S}$ latitude), inhabiting a 
variety of habitats in the Altoandina, Puna, and Prepuna biogeographical provinces (sensu Cabrera $\&$ Willink, 1980). These highland ecosystems are considered highly sensitive to climatic change and global warming (Halloy \& Mark, 2003; Gonzales, 2009; IPCC, 2014; Cuesta et al., 2017). Furthermore, the restricted altitudinal range that many species occupy within these highland ecosystems, enhances their vulnerability to climatic change (Urban, 2015). On the other hand, endemics of this family are also present in lowlands of Patagonia and Central-Eastern Argentina [e.g., Chilocardamum patagonicum, Lepidium parodii, Lepidium hickenii, Mostacillastrum subscandens, Mostacillastrum ventanense, Physaria lateralis, and Trichotolinum deserticola] (Al-Shehbaz, 2012a). These areas are generally exposed to high human impact and land-use pressures, which can have a direct effect on the habitat degradation and biodiversity loss (Grau et al., 2005; Paruelo et al., 2006; Volante et al., 2012; Newbold et al., 2015; Vallejos et al., 2015). Additionally, several Argentinean endemic genera, such as Delphinophytum Speg., Litodraba Boelcke, and Zuloagocardamum Salariato \& AlShehbaz, are micro-endemics with very restricted distribution ranges (Al-Shehbaz, 2012a; Salariato $\&$ Al-Shehbaz, 2014). Therefore, disturbances of their habitats expose these species to a high level of vulnerability (Gaston, 1994).

Based on these observations for the Brassicaceae family, and since urgent conservation strategies need to be implemented in order to preserve the native flora of Argentina (Morea, 2014), here we explored the geographic distribution of Argentinean crucifers using georeferenced species distributional data, to preliminary classify the species according to the IUCN threat categories, and analyzing patterns of richness, endemism, and threat in the different ecoregions and biomes of the country. Furthermore, elevation, annual mean temperature, annual precipitation, and aridity were compared for endemic vs. non-endemic and threatened vs. nonthreatened species. Geographical range maps for all Argentinean native crucifers are also provided. This work seeks to contribute to the knowledge of the geographical distribution of the Argentinean flora and its conservation, complementing the Brassicaceae treatment of the Argentinean Flora (Al-Shehbaz, 2012a).

\section{MATERIALS AND METHODS}

\section{Species occurrence data and threat assessments}

Extensive botanical surveys of the Brassicaceae family were conducted in Argentina by the authors between 2008 and 2020. Collection sites were geographically referenced using the WGS 84 geodetic datum, and specimens collected were deposited in SI, with duplicates in several herbaria (herbarium acronyms following Thiers, 2020). Additionally, we studied specimens deposited in different herbaria, mainly from BA, BAA, BAB, BCRU, CORD, CTES, LIL, LP, MERL, MO, $\mathrm{SF}$, and SI. Based on the most recent taxonomic treatments and phylogenetic analyses (Al-Shehbaz, 2012a; Salariato et al., 2013a; 2014; 2015a; 2015b; 2018; 2019; 2020a; 2020b; 2020c; Salariato \& Al-Shehbaz, 2014; Salariato \& Zuloaga, 2015), we included 162 taxa representing 160 species and two subspecies, corresponding to Menonvillea scapigera and Xerodraba patagonica, since the differentiation of these subspecies with the type subspecies has been supported by both morphological and molecular data (Salariato et al. 2012, 2015a). Records were also checked in the Flora of Argentina (Al-Shehbaz, 2012a) and the Documenta Florae Australis database (http:// www.darwin.edu.ar/iris) (last accessed March 2020), which represents the most up-to-date and comprehensive database of the Argentinean Flora (Zuloaga et al., 2019). For species and subspecies author names see Table 1. Occurrences were mapped using QGIS v3.14.16 'Pi' (Quantum GIS Development Team, 2016) for visual inspection, and in cases of specimens with no GPS coordinates but exact locality names, records were georeferenced using Google Earth Pro v7.3.3.7786 (https://www. google.com/intl/en/earth/). We obtained 5258 data points corresponding to 35 genera and 162 species/ subspecies present in Argentina (species occurrences: $\min =1, \max =285$, mean $=32)$ (Fig. S1, supplementary material).

Preliminary threat assessments for Argentinean species were based on the IUCN Red List categories and criteria v3.1 (IUCN, 2012) following the IUCN guidelines v14 (IUCN, 2019). Conservation status were determined under criterion $\mathrm{B}$, which is based on geographic ranges (IUCN, 2012; 2019) 
and suitable for estimating conservation status when the distribution of taxa is only known from georeferenced herbarium specimens with limited information on abundance and potential continuing decline (Schatz, 2002; Nic Lughadha et al., 2018). For each species we used its extent of occurrence (EOO) (subcriterion B1; IUCN, 2019) calculated with a minimum convex polygon around occurrence points and clipped to the extent of Argentina. Area of occupancy (AOO) (subcriteria B2) was not used for categorization because it can lead to overestimation of extinction risk (when herbarium specimens are used occurrences are rarely sufficient to support calculation of AOO using the recommended 4 $\mathrm{km}^{2}$ grid cell; Nic Lughadha et al., 2018; IUCN, 2019). However, for species with less than three unique occurrences, for which EOO cannot be computed, AOO was reported. To estimate the number of locations for each species (condition "a", a location is defined as a geographically or ecologically distinct area in which a single threat can rapidly affect all individuals of the taxon present; IUCN, 2019) we registered the number of cells of 0.1 arc-degrees $\left(\sim 100 \mathrm{~km}^{2}\right)$ occupied by the occurrences. For condition " $b$ " we evaluated vulnerability and potential deterioration of the habitat where species grow (case iii), considering ecoregions and biomes, and using data from field observations, herbarium labels, and literature, to characterize the environment where they inhabit (Brown et al., 2006; Brooks et al., 2006; 2015; INTA, 2009; Chehébar et al., 2013; Morello et al., 2018; Nanni et al., 2020). All IUCN parameters needed for assessments of taxa under criterion $\mathrm{B}$ were calculated using the package ConR v1.3.0 (Dauby et al., 2017; Dauby, 2020) implemented in R v4.0.2 (R Core Team, 2020).

\section{Spatial analyses}

To study the geographic patterns of richness and threat in a biogeographic context, we analyzed the distribution of four variables: a) number of species (richness), b) number of threatened species (considering as threatened species all species under $\mathrm{CR}, \mathrm{EN}$, and VU categories), c) number of endemic species (species only present in Argentina), and d) number of threatened endemic species. First, we registered numbers of these variables for political provinces, ecoregions, and biomes of Argentina; these last two eco-geographical classifications sensu Olson et al. (2001) and included in a shapefile provided by the WWF (https://www.worldwildlife. org/publications/terrestrial-ecoregions-of-the-world). Although these values were calculated at family level, they were also estimated for the different tribes (Al-Shehbaz, 2012b). In addition, the similarity of ecoregions in terms of their species composition was studied using Jaccard distances and agglomerative hierarchical clustering with the unweighted pairgroup average (UPGMA) algorithm in the R packages vegan v2.5-6 (Oksanen et al., 2019) and cluster v2.1.0 (Maechler et al., 2019).

Alternatively, we explored spatial patterns for the four variables (richness, threat, endemics, and threatened endemics). Because the use of small cells would result in a finer and more detailed resolution, but at the same time increase the number of artificially empty cells where species occur but have not been recorded (Linder, 2001), we used grids at two different cell sizes, 1 and 0.5 arc-degrees (approx. $100 \times 100 \mathrm{~km}$ and $50 \times$ $50 \mathrm{~km}$, respectively), to explore both distribution patterns at different scales and the robustness of the results to changes in the grid size. Analyses were conducted using the $\mathrm{R}$ packages raster v3.1.5 (Hijmans, 2020) and sp v1.4.2 (Pebesma \& Bivand, 2005; Bivand et al., 2013). The protection status of the hottest cells was evaluated overlaying shapefiles of the protected areas in Argentina provided by the Biodiversity Information System of the National Parks Administration (SIB-APN) (https://sib.gob.ar/cartografia).

We also analyzed differences in elevation, annual mean temperature (BIO1), annual precipitation (BIO12), and aridity (AI) for endemic vs. non-endemic and threatened vs. non-threatened species. These variables can represent limiting climatic factors that constrain species distributions in the face of global warming and desertification (Jezkova \& Wiens, 2016). After removing occurrences closer to 30 arc-seconds $(\sim 1 \mathrm{~km})$, we extracted values of $\mathrm{BIO} 1$ and $\mathrm{BIO} 12$ for each occurrence point from the CHELSA v1.2 climatic dataset (Karger et al., 2017a; 2017b; https://chelsaclimate.org/) at a resolution of 30 arc-seconds $\left(\sim 1 \mathrm{~km}^{2}\right)$, while values of the annual aridity (AI) were obtained from the CGIARCS v2 database (Trabucco \& Zomer, 2019) at the same resolution. 
Elevation data was extracted from specimen vouchers and by georeferencing. Values obtained for each variable were then used to calculate the mean of each species, and the Mann-Whitney U test was conducted to assess for differences between (1) endemic vs. non-endemic species and (2) threatened vs. non-threatened species, for elevation, BIO1, BIO12, and IA. Despite the fact these variables may exhibit phylogenetic signal, this is an explicitly nonphylogenetic test, since rather than an evolutionary signal, we are interested in whether the association exists at time zero given current conditions.

Finally, environments where species grow were categorized following the climate classification scheme for Aridity Index values (UNEP, 1997) $(<$ 0.03: hyper Arid, $0.03-0.2$ : arid, $0.2-0.5$ : semi-arid. $0.5-0.65$ : dry sub-humid, $>0.65$ humid). For each species we used the AI values corresponding to the $5^{\text {th }}$ and $95^{\text {th }}$ percentile to capture the niche breadth.

\section{RESULTS}

We compiled a total of 5258 presence records of Brassicaceae for Argentina, representing eleven tribes, 35 genera, 160 species, and two subspecies (Table 1). After the preliminary IUCN threat categorization we classified 97 species $(60 \%)$ under Least Concern (LC), 7 (4\%) as Near Threatened (NT), 19 (12\%) as Vulnerable (VU), 25 (15\%) as Endangered (EN), and $14(9 \%)$ as Critically Endangered (CR) (Table 1, Fig. 1); counting a total of 58 species under threatened categories (36\%) (geographic range maps for each species and the rationale for the threat categories are supplied in Appendix $1 \mathrm{http}: / /$ www.ojs.darwin.edu.ar/index.php/darwiniana/ article/view/922/1200 and Appendix 2 http://www. ojs.darwin.edu.ar/index.php/darwiniana/article/ view/922/1201, respectively). Argentinian crucifers were present in all political provinces, ecoregions, and biomes (Tables S1, S2, and S3, Supplementary Material), with 58 species $(\sim 36 \%)$ and eight genera endemic to Argentina (Chilocardamum O. E. Schulz, Delpinophytum, Lithodraba, Parodiodoxa O. E. Schulz, Petroravenia Al-Shehbaz, Phlebolobium O. E. Schulz, Trichotolinum O. E. Schulz, and Zuloagocardamum) (Fig. 2) (Table 1). Of these 58 Argentinean endemics, 33 species ( 57\%) are classified in some threat category (Table 1).
When we analyze the number of endemics and threatened species by tribe, Thelypodieae presented the highest values, followed by Lepidieae and Eudemeae (Table S4, Supplementary Material).

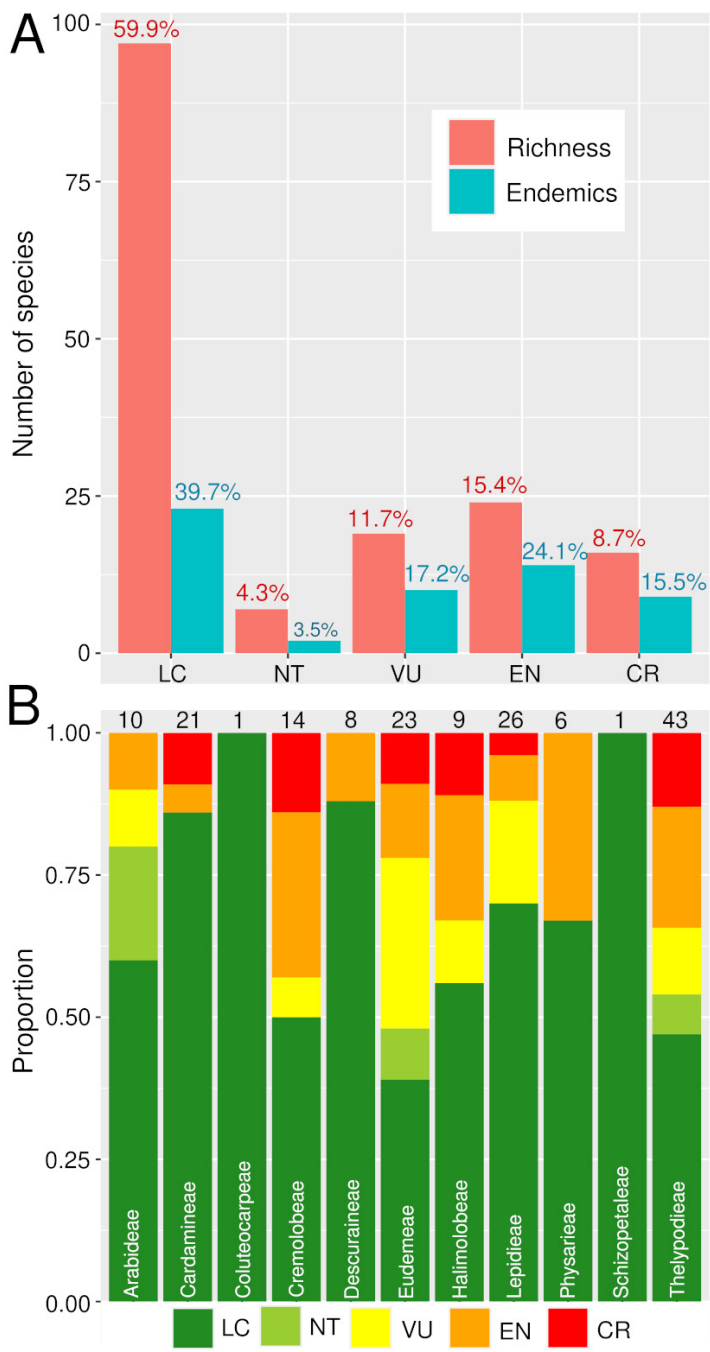

Fig. 1. Preliminary threat assessments for Argentinean crucifers were based on the IUCN red list categories and criteria. A, Barplots showing number of species (red) and endemics (blue) for each IUCN category. Numbers above the boxes correspond to the percentage of species included in each category with respect to the total number of species (red) and endemics (blue). B, Barplots showing the proportion of species of each tribe classified in the different categories. Numbers above the boxes correspond to the total number of species for each tribe. Color version at http://www.ojs.darwin.edu.ar/ index.php/darwiniana/article/view/922/1199 
Table 1. Preliminary threat assessments for Argentinean species based on the IUCN red list categories and criteria. EOO (extent of occurrence) was calculated with a minimum convex polygon around occurrence points and clipped to the extent of Argentina. *For species with less than three unique occurrences (EOO cannot be computed) area of occupancy $(\mathrm{AOO})$ was reported. For the geographical ranges: $\mathrm{AR}=$ Argentina, $\mathrm{BO}=$ Bolivia, $\mathrm{BR}=\mathrm{Brazil}, \mathrm{CH}=\mathrm{Chile}, \mathrm{PA}=$ Paraguay, $\mathrm{PE}=$ Peru, UR= Uruguay, $+=$ other countries in northern South America. Geographic range maps for each species and the rationale for the preliminary threat categories are supplied in Appendix 1 and Appendix 2, respectively.

\begin{tabular}{|c|c|c|c|c|c|}
\hline Species & Tribe & Status & $\begin{array}{r}\text { EOO } \\
\left(\mathbf{k m}^{2}\right)\end{array}$ & $\begin{array}{l}\mathrm{N}^{\circ} \text { of locations } \\
\text { based in } \\
0.1^{\circ} \text { cells }\end{array}$ & $\begin{array}{l}\text { IUCN category } \\
\text { based in } \\
\text { B1ab(iii) criteria }\end{array}$ \\
\hline $\begin{array}{l}\text { Alshehbazia friesii (O.E. Schulz) Salariato, } \\
\text { Zuloaga \& Al-Shehbaz }\end{array}$ & Eudemeae & $\begin{array}{l}\text { native }(\mathrm{AR}, \mathrm{BO} \\
\quad \mathrm{CH}, \mathrm{PE})\end{array}$ & $4^{*}$ & 1 & $\mathrm{CR}$ \\
\hline $\begin{array}{l}\text { Alshehbazia hauthalii (Gilg \& Muschl.) } \\
\text { Salariato \& Zuloaga }\end{array}$ & Eudemeae & native $(\mathrm{AR}, \mathrm{CH})$ & 4488 & 4 & $\mathrm{EN}$ \\
\hline $\begin{array}{l}\text { Alshehbazia werdermannii (O.E. Schulz) } \\
\text { Salariato, Zuloaga \& Al-Shehbaz }\end{array}$ & Eudemeae & $\begin{array}{l}\text { native }(\mathrm{AR}, \mathrm{CH} \\
\mathrm{PE})\end{array}$ & 32012 & 12 & NT \\
\hline $\begin{array}{l}\text { Aschersoniodoxa cachensis (Speg.) Al- } \\
\text { Shehbaz }\end{array}$ & Eudemeae & $\begin{array}{l}\text { native }(\mathrm{AR}, \mathrm{BO} \\
\mathrm{PE})\end{array}$ & 19441 & 4 & VU \\
\hline Brayopsis calycina (Desv.) Gilg \& Muschl. & Eudemeae & $\begin{array}{l}\text { native (AR, } \mathrm{BO} \\
\mathrm{PE})\end{array}$ & 21910 & 8 & NT \\
\hline Brayopsis monimocalyx O.E. Schulz & Eudemeae & $\begin{array}{l}\text { native (AR, } \mathrm{BO} \\
\mathrm{PE})\end{array}$ & 41104 & 11 & $\mathrm{LC}$ \\
\hline Cardamine bonariensis Pers. & Cardamineae & $\begin{array}{c}\text { native }(\mathrm{AR}, \mathrm{BO}, \\
\mathrm{BR}, \mathrm{CH}, \mathrm{PA}, \mathrm{PE}, \\
\mathrm{UR},+)\end{array}$ & 2271211 & 59 & $\mathrm{LC}$ \\
\hline Cardamine chenopodiifolia Pers. & Cardamineae & $\begin{array}{l}\text { native (AR, BO, } \\
\mathrm{BR}, \mathrm{CH}, \mathrm{PA}, \mathrm{UR})\end{array}$ & 483466 & 39 & $\mathrm{LC}$ \\
\hline Cardamine chilensis DC. & Cardamineae & native $(\mathrm{AR}, \mathrm{CH})$ & 32327 & 13 & $\mathrm{LC}$ \\
\hline Cardamine cordata Barnéoud & Cardamineae & native $(\mathrm{AR}, \mathrm{CH})$ & 162683 & 47 & $\mathrm{LC}$ \\
\hline Cardamine geraniifolia (Poir.) DC. & Cardamineae & native $(\mathrm{AR}, \mathrm{CH})$ & 38220 & 12 & $\mathrm{LC}$ \\
\hline Cardamine glacialis (G. Forst.) DC. & Cardamineae & native (AR, $\mathrm{CH})$ & 218392 & 48 & $\mathrm{LC}$ \\
\hline Cardamine rostrata Griseb. & Cardamineae & native $(\mathrm{AR}, \mathrm{CH})$ & $4^{*}$ & 1 & $\mathrm{CR}$ \\
\hline Cardamine tenuirostris Hook. \& Arn. & Cardamineae & native (AR, $\mathrm{CH})$ & 27998 & 19 & $\mathrm{LC}$ \\
\hline Cardamine tuberosa DC. & Cardamineae & native $(\mathrm{AR}, \mathrm{CH})$ & $4 *$ & 1 & $\mathrm{CR}$ \\
\hline Cardamine variabilis Phil. & Cardamineae & native $(\mathrm{AR}, \mathrm{CH})$ & 122683 & 35 & $\mathrm{LC}$ \\
\hline Cardamine volckmannii Phil. & Cardamineae & native (AR, $\mathrm{CH})$ & 47359 & 13 & $\mathrm{LC}$ \\
\hline Cardamine vulgaris Phil. & Cardamineae & native $(\mathrm{AR}, \mathrm{CH})$ & 46287 & 30 & $\mathrm{LC}$ \\
\hline $\begin{array}{l}\text { Chilocardamum castellanosii (O.E. Schulz) } \\
\text { Al-Shehbaz }\end{array}$ & Thelypodieae & endemic (AR) & 175551 & 6 & $\mathrm{LC}$ \\
\hline $\begin{array}{l}\text { Chilocardamum longistylum (Romanczuk) } \\
\text { Al-Shehbaz }\end{array}$ & Thelypodieae & endemic (AR) & $8^{*}$ & 2 & EN \\
\hline $\begin{array}{l}\text { Chilocardamum onuridifolium (Ravenna) } \\
\text { Al-Shehbaz }\end{array}$ & Thelypodieae & endemic (AR) & 702 & 3 & $\mathrm{EN}$ \\
\hline $\begin{array}{l}\text { Chilocardamum patagonicum (Speg.) O.E. } \\
\text { Schulz }\end{array}$ & Thelypodieae & endemic (AR) & 237873 & 19 & $\mathrm{LC}$ \\
\hline Cremolobus chilensis (Lag. ex DC.) DC. & Cremolobeae & $\begin{array}{l}\text { native }(\mathrm{AR}, \mathrm{BO} \\
\quad \mathrm{CH}, \mathrm{PE})\end{array}$ & 3613 & 3 & $\mathrm{EN}$ \\
\hline Delpinophytum patagonicum (Speg.) Speg. & Eudemeae & endemic (AR) & 16201 & 3 & VU \\
\hline $\begin{array}{l}\text { Descurainia antarctica (E. Fourn.) O.E. } \\
\text { Schulz }\end{array}$ & Descuraineae & native $(\mathrm{AR}, \mathrm{CH})$ & 606947 & 34 & $\mathrm{LC}$ \\
\hline Descurainia depressa (Phil.) Prantl ex Reiche & Descuraineae & $\begin{array}{l}\text { native }(\mathrm{AR}, \mathrm{BO} \\
\mathrm{CH}, \mathrm{PE})\end{array}$ & 720835 & 48 & $\mathrm{LC}$ \\
\hline $\begin{array}{l}\text { Descurainia erodiifolia (Phil.) Prantl ex } \\
\text { Reiche }\end{array}$ & Descuraineae & $\begin{array}{l}\text { native }(\mathrm{AR}, \mathrm{BO} \\
\mathrm{CH}, \mathrm{UR})\end{array}$ & 2177834 & 172 & $\mathrm{LC}$ \\
\hline $\begin{array}{l}\text { Descurainia myriophylla (Willd. ex DC.) R.E. } \\
\text { Fr. }\end{array}$ & Descuraineae & $\begin{array}{l}\text { native }(\mathrm{AR}, \mathrm{BO} \\
\quad \mathrm{CH}, \mathrm{PE},+)\end{array}$ & 101134 & 25 & $\mathrm{LC}$ \\
\hline
\end{tabular}


Table 1. (Continuation).

\begin{tabular}{|c|c|c|c|c|c|}
\hline Species & Tribe & Status & $\begin{array}{r}\text { EOO } \\
\left(\mathbf{k m}^{2}\right)\end{array}$ & $\begin{array}{l}\mathrm{N}^{0} \text { of locations } \\
\text { based in } \\
0.1^{\circ} \text { cells }\end{array}$ & $\begin{array}{l}\text { IUCN category } \\
\text { based in } \\
\text { B1ab(iii) criteria }\end{array}$ \\
\hline Descurainia nuttallii (Colla) O.E. Schulz & Descuraineae & native $(\mathrm{AR}, \mathrm{CH})$ & 1123519 & 30 & LC \\
\hline $\begin{array}{l}\text { Descurainia pimpinellifolia (Barnéoud) O.E. } \\
\text { Schulz }\end{array}$ & Descuraineae & native $(\mathrm{AR}, \mathrm{CH})$ & 506661 & 72 & $\mathrm{LC}$ \\
\hline Descurainia stricta (Phil.) Prantl ex Reiche & Descuraineae & $\begin{array}{c}\text { native }(\mathrm{AR}, \mathrm{BO} \\
\mathrm{CH}, \mathrm{PE})\end{array}$ & 142216 & 27 & $\mathrm{LC}$ \\
\hline $\begin{array}{l}\text { Dictyophragmus punensis (Romanczuk) Al- } \\
\text { Shehbaz }\end{array}$ & Thelypodieae & endemic (AR) & 1893 & 6 & VU \\
\hline Draba burkartiana O.E. Schulz & Arabideae & endemic (AR) & 6864 & 7 & VU \\
\hline Draba funiculosa Hook. f. & Arabideae & native $(\mathrm{AR}, \mathrm{CH})$ & 26448 & 9 & NT \\
\hline Draba gilliesii Hook. \& Arn. & Arabideae & native $(\mathrm{AR}, \mathrm{CH})$ & 91955 & 45 & $\mathrm{LC}$ \\
\hline Draba lapaziana Al-Shehbaz & Arabideae & native $(\mathrm{AR}, \mathrm{BO})$ & 4915 & 3 & EN \\
\hline Draba loayzana Al-Shehbaz & Arabideae & native (AR, BO) & 23452 & 4 & NT \\
\hline Draba macleanii Hook. f. & Arabideae & $\begin{array}{l}\text { native }(\mathrm{AR}, \mathrm{BO} \\
\mathrm{CH}, \mathrm{PE})\end{array}$ & 60529 & 11 & $\mathrm{LC}$ \\
\hline Draba magellanica Lam. & Arabideae & native $(\mathrm{AR}, \mathrm{CH})$ & 909958 & 69 & $\mathrm{LC}$ \\
\hline Draba pusilla F. Phil. & Arabideae & native $(\mathrm{AR}, \mathrm{CH})$ & 314047 & 18 & $\mathrm{LC}$ \\
\hline Draba tucumanensis O.E. Schulz & Arabideae & endemic (AR) & 126037 & 17 & $\mathrm{LC}$ \\
\hline $\begin{array}{l}\text { Exhalimolobos burkartii (Romanczuk \& } \\
\text { Boelcke) Al-Shehbaz \& C.D. Bailey }\end{array}$ & Halimolobeae & endemic (AR) & 9208 & 3 & VU \\
\hline $\begin{array}{l}\text { Exhalimolobos pazense (Rusby) Al-Shehbaz } \\
\text { \& C.D. Bailey }\end{array}$ & Halimolobeae & $\begin{array}{l}\text { native (AR, } \mathrm{BO} \\
\mathrm{PE})\end{array}$ & 178412 & 16 & $\mathrm{LC}$ \\
\hline $\begin{array}{l}\text { Exhalimolobos weddellii (E. Fourn.) Al- } \\
\text { Shehbaz \& C.D. Bailey }\end{array}$ & Halimolobeae & $\begin{array}{l}\text { native }(\mathrm{AR}, \mathrm{BO} \\
\mathrm{PE}, \mathrm{UR})\end{array}$ & 1275817 & 129 & $\mathrm{LC}$ \\
\hline Lepidium argentinum Thell. & Lepidieae & endemic (AR) & 150678 & 31 & $\mathrm{LC}$ \\
\hline Lepidium auriculatum Regel \& Körn. & Lepidieae & $\begin{array}{c}\text { native (AR, BO, } \\
\text { BR, CH, PA, } \\
\text { UR) }\end{array}$ & 2442239 & 124 & $\mathrm{LC}$ \\
\hline Lepidium boelckeanum Prina & Lepidieae & endemic (AR) & 124937 & 9 & $\mathrm{LC}$ \\
\hline Lepidium boelckei Al-Shehbaz & Lepidieae & endemic (AR) & 53818 & 12 & $\mathrm{LC}$ \\
\hline Lepidium bonariense $\mathrm{L}$. & Lepidieae & $\begin{array}{c}\text { native (AR, BO, } \\
\text { BR, CH, PA, } \\
\text { UR) }\end{array}$ & 1881643 & 202 & $\mathrm{LC}$ \\
\hline Lepidium burkartii Boelcke & Lepidieae & endemic (AR) & 943 & 3 & EN \\
\hline Lepidium depressum Thell. & Lepidieae & $\begin{array}{c}\text { native (AR, BO, } \\
\mathrm{PE})\end{array}$ & 106762 & 10 & $\mathrm{LC}$ \\
\hline Lepidium didymum $\mathrm{L}$. & Lepidieae & $\begin{array}{c}\text { native (AR, BO, } \\
\text { BR, CH, PA, PE, } \\
\text { UR) }\end{array}$ & 2545977 & 108 & $\mathrm{LC}$ \\
\hline Lepidium filisegmentum C.L. Hitchc. & Lepidieae & native $(\mathrm{AR}, \mathrm{CH})$ & 450829 & 28 & $\mathrm{LC}$ \\
\hline Lepidium gracile (Chodat \& Hassl.) Boelcke & Lepidieae & native (AR, PA) & 641026 & 30 & $\mathrm{LC}$ \\
\hline Lepidium hickenii Al-Shehbaz & Lepidieae & endemic (AR) & 19840 & 4 & VU \\
\hline Lepidium jujuyanum Al-Shehbaz & Lepidieae & endemic (AR) & 11208 & 6 & VU \\
\hline Lepidium meyenii Walp. & Lepidieae & $\begin{array}{c}\text { native }(\mathrm{AR}, \mathrm{BO} \\
\mathrm{CH}, \mathrm{PE})\end{array}$ & 119411 & 32 & $\mathrm{LC}$ \\
\hline Lepidium myrianthum Phil. & Lepidieae & native $(\mathrm{AR}, \mathrm{CH})$ & 715535 & 46 & $\mathrm{LC}$ \\
\hline Lepidium parodii Thell. & Lepidieae & endemic (AR) & 480463 & 41 & $\mathrm{LC}$ \\
\hline Lepidium pedersenii Al-Shehbaz & Lepidieae & native (AR, PA) & 352570 & 31 & $\mathrm{LC}$ \\
\hline Lepidium pseudodidymum Thell. ex Druce & Lepidieae & native $(\mathrm{AR}, \mathrm{CH})$ & 65674 & 12 & $\mathrm{LC}$ \\
\hline Lepidium rahmeri Phil. & Lepidieae & native $(\mathrm{AR}, \mathrm{CH})$ & 129530 & 23 & $\mathrm{LC}$ \\
\hline Lepidium reichei Phil. ex Reiche & Lepidieae & native (AR, $\mathrm{CH})$ & 221 & 3 & EN \\
\hline
\end{tabular}


Table 1. (Continuation).

\begin{tabular}{|c|c|c|c|c|c|}
\hline Species & Tribe & Status & $\begin{array}{l}\text { EOO } \\
\left(\mathbf{k m}^{2}\right)\end{array}$ & $\begin{array}{l}\mathrm{N}^{\circ} \text { of locations } \\
\text { based in } \\
0.1^{\circ} \text { cells }\end{array}$ & $\begin{array}{l}\text { IUCN category } \\
\text { based in } \\
\text { B1ab(iii) criteria }\end{array}$ \\
\hline Lepidium rhytidocarpum (Hook.) Al-Shehbaz & Lepidieae & native (AR, UR) & 280893 & 11 & $\mathrm{LC}$ \\
\hline Lepidium santacruzensis Al-Shehbaz & Lepidieae & endemic (AR) & $4^{*}$ & 1 & $\mathrm{CR}$ \\
\hline Lepidium serratum (Poir.) Al-Shehbaz & Lepidieae & native (AR, UR) & 19582 & 3 & VU \\
\hline Lepidium spicatum Desv. & Lepidieae & native $(\mathrm{AR}, \mathrm{CH})$ & 1680691 & 58 & $\mathrm{LC}$ \\
\hline Lepidium stuckertianum (Thell.) Boelcke & Lepidieae & endemic (AR) & 917374 & 65 & $\mathrm{LC}$ \\
\hline Lepidium tandilense Boelcke & Lepidieae & native (AR, UR) & 85057 & 7 & $\mathrm{LC}$ \\
\hline $\begin{array}{l}\text { Lithodraba mendocinensis (Hauman) } \\
\text { Boelcke }\end{array}$ & Lepidieae & endemic (AR) & 16250 & 10 & VU \\
\hline Mancoa foliosa (Wedd.) O.E. Schulz & Halimolobeae & native (AR, BO) & $8^{*}$ & 2 & EN \\
\hline Mancoa hispida Wedd. & Halimolobeae & $\begin{array}{l}\text { native }(\mathrm{AR}, \mathrm{BO} \\
\mathrm{CH}, \mathrm{PE})\end{array}$ & 100653 & 24 & $\mathrm{LC}$ \\
\hline Mancoa venturii Al-Shehbaz & Halimolobeae & native (AR, $\mathrm{PE})$ & 21618 & 17 & $\mathrm{LC}$ \\
\hline Menonvillea cicatricosa (Phil.) Rollins & Cremolobeae & native $(\mathrm{AR}, \mathrm{CH})$ & 12 & 1 & $\mathrm{CR}$ \\
\hline Menonvillea comberi Sandwith & Cremolobeae & native $(\mathrm{AR}, \mathrm{CH})$ & 744 & 3 & EN \\
\hline $\begin{array}{l}\text { Menonvillea cuneata (Gillies \& Hook.) } \\
\text { Rollins }\end{array}$ & Cremolobeae & native $(\mathrm{AR}, \mathrm{CH})$ & 64328 & 59 & $\mathrm{LC}$ \\
\hline Menonvillea famatinensis (Boelcke) Rollins & Cremolobeae & endemic (AR) & 26 & 3 & EN \\
\hline Menonvillea nordenskjoeldii (Dusén) Rollins & Cremolobeae & native $(\mathrm{AR}, \mathrm{CH})$ & 40410 & 26 & $\mathrm{LC}$ \\
\hline Menonvillea patagonica Speg. & Cremolobeae & endemic (AR) & 74268 & 12 & $\mathrm{LC}$ \\
\hline Menonvillea rigida Rollins & Cremolobeae & endemic (AR) & 4831 & 9 & VU \\
\hline $\begin{array}{l}\text { Menonvillea scapigera (Phil.) Rollins longipes } \\
\text { (Rollins) Prina }\end{array}$ & Cremolobeae & native $(\mathrm{AR}, \mathrm{CH})$ & 117130 & 40 & $\mathrm{LC}$ \\
\hline $\begin{array}{l}\text { Menonvillea scapigera (Phil.) Rollins } \\
\text { scapigera }\end{array}$ & Cremolobeae & endemic (AR) & 27187 & 21 & $\mathrm{LC}$ \\
\hline $\begin{array}{l}\text { Menonvillea spathulata (Gillies \& Hook.) } \\
\text { Rollins }\end{array}$ & Cremolobeae & native $(\mathrm{AR}, \mathrm{CH})$ & 36784 & 18 & $\mathrm{LC}$ \\
\hline Menonvillea virens (Phil.) Rollins & Cremolobeae & native $(\mathrm{AR}, \mathrm{CH})$ & 118061 & 22 & $\mathrm{LC}$ \\
\hline Menonvillea zuloagaensis Al-Shehbaz & Cremolobeae & endemic (AR) & $4^{*}$ & 1 & $\mathrm{CR}$ \\
\hline $\begin{array}{l}\text { Mostacillastrum ameghinoi (Speg.) O.E. } \\
\text { Schulz }\end{array}$ & Thelypodieae & endemic (AR) & 36126 & 3 & NT \\
\hline Mostacillastrum andinum (Phil.) Al-Shehbaz & Thelypodieae & native $(\mathrm{AR}, \mathrm{CH})$ & 59598 & 15 & $\mathrm{LC}$ \\
\hline $\begin{array}{l}\text { Mostacillastrum carolinense (Scappini, C.A. } \\
\text { Bianco \& Prina) Al-Shehbaz }\end{array}$ & Thelypodieae & endemic (AR) & $4^{*}$ & 1 & $\mathrm{CR}$ \\
\hline Mostacillastrum commune (Speg.) Al-Shehbaz & Thelypodieae & native $(\mathrm{AR}, \mathrm{CH})$ & 490735 & 23 & $\mathrm{LC}$ \\
\hline $\begin{array}{l}\text { Mostacillastrum dianthoides (Phil.) Al- } \\
\text { Shehbaz }\end{array}$ & Thelypodieae & $\begin{array}{c}\text { native }(\mathrm{AR}, \mathrm{CH} \\
\mathrm{PE})\end{array}$ & 4762 & 5 & $\mathrm{EN}$ \\
\hline Mostacillastrum hunzikeri Al-Shehbaz & Thelypodieae & endemic (AR) & 219 & 3 & $\mathrm{EN}$ \\
\hline $\begin{array}{l}\text { Mostacillastrum leptocarpum (Hook. \& Arn.) } \\
\text { Al-Shehbaz }\end{array}$ & Thelypodieae & native $(\mathrm{AR}, \mathrm{CH})$ & 38035 & 16 & $\mathrm{LC}$ \\
\hline $\begin{array}{l}\text { Mostacillastrum orbignyanum (E. Fourn.) } \\
\text { Al-Shehbaz }\end{array}$ & Thelypodieae & native (AR, BO) & 497571 & 61 & $\mathrm{LC}$ \\
\hline Mostacillastrum saltaensis Al-Shehbaz & Thelypodieae & endemic (AR) & $4 *$ & 1 & $\mathrm{CR}$ \\
\hline $\begin{array}{l}\text { Mostacillastrum stenophyllum (Gillies ex } \\
\text { Hook. \& Arn.) O.E. Schulz }\end{array}$ & Thelypodieae & endemic (AR) & 195357 & 50 & $\mathrm{LC}$ \\
\hline $\begin{array}{l}\text { Mostacillastrum subscandens (Speg.) Al- } \\
\text { Shehbaz }\end{array}$ & Thelypodieae & endemic (AR) & 92038 & 5 & $\mathrm{LC}$ \\
\hline $\begin{array}{l}\text { Mostacillastrum ventanense (Boelcke) Al- } \\
\text { Shehbaz }\end{array}$ & Thelypodieae & endemic (AR) & 116 & 3 & EN \\
\hline $\begin{array}{l}\text { Neuontobotrys choiquense (Romanczuk) } \\
\text { Al-Shehbaz }\end{array}$ & Thelypodieae & endemic (AR) & 5932 & 3 & VU \\
\hline
\end{tabular}


Table 1. (Continuation).

\begin{tabular}{|c|c|c|c|c|c|}
\hline Species & Tribe & Status & $\begin{array}{r}\text { EOO } \\
\left(\mathbf{k m}^{2}\right)\end{array}$ & $\begin{array}{l}\mathrm{N}^{\circ} \text { of locations } \\
\text { based in } \\
0.1^{\circ} \text { cells }\end{array}$ & $\begin{array}{l}\text { IUCN category } \\
\text { based in } \\
\text { B1ab(iii) criteria }\end{array}$ \\
\hline $\begin{array}{l}\text { Neuontobotrys frutescens (Gillies ex Hook. \& } \\
\text { Arn.) Al-Shehbaz }\end{array}$ & Thelypodieae & endemic (AR) & 114179 & 32 & $\mathrm{LC}$ \\
\hline $\begin{array}{l}\text { Neuontobotrys mendocina (Romanczuk) Al- } \\
\text { Shehbaz }\end{array}$ & Thelypodieae & native $(\mathrm{AR}, \mathrm{CH})$ & 9021 & 4 & VU \\
\hline Neuontobotrys polyphylla (Phil.) Al-Shehbaz & Thelypodieae & native $(\mathrm{AR}, \mathrm{CH})$ & $4^{*}$ & 1 & $\mathrm{CR}$ \\
\hline $\begin{array}{l}\text { Neuontobotrys robusta (Chodat \& Wilczek) } \\
\text { Al-Shehbaz }\end{array}$ & Thelypodieae & endemic (AR) & 41415 & 9 & $\mathrm{LC}$ \\
\hline Neuontobotrys tarapacana (Phil.) Al-Shehbaz & Thelypodieae & native $(\mathrm{AR}, \mathrm{CH})$ & 44399 & 19 & $\mathrm{LC}$ \\
\hline Noccaea magellanica (Comm. ex Poir.) Holub & Coluteocarpeae & native $(\mathrm{AR}, \mathrm{CH})$ & 757307 & 104 & $\mathrm{LC}$ \\
\hline Onuris alismatifolia Gilg ex Skottsb. & Eudemeae & native $(\mathrm{AR}, \mathrm{CH})$ & 5002 & 8 & VU \\
\hline Onuris graminifolia Phil. & Eudemeae & native $(\mathrm{AR}, \mathrm{CH})$ & 471184 & 49 & $\mathrm{LC}$ \\
\hline $\begin{array}{l}\text { Onuris hatcheriana (Gilg ex Macloskie) Gilg } \\
\& \text { Muschl. }\end{array}$ & Eudemeae & native $(\mathrm{AR}, \mathrm{CH})$ & 14416 & 5 & VU \\
\hline Onuris papillosa O.E. Schulz & Eudemeae & native $(\mathrm{AR}, \mathrm{CH})$ & 23373 & 14 & $\mathrm{LC}$ \\
\hline Onuris spegazziniana Gilg \& Muschl. & Eudemeae & native $(\mathrm{AR}, \mathrm{CH})$ & 183032 & 11 & $\mathrm{LC}$ \\
\hline Parodiodoxa chionophila (Speg.) O.E. Schulz & Thelypodieae & endemic (AR) & 58561 & 14 & $\mathrm{LC}$ \\
\hline Pennellia boliviensis (Muschl.) Al-Shehbaz & Halimolobeae & native $(\mathrm{AR}, \mathrm{BO})$ & 110188 & 20 & $\mathrm{LC}$ \\
\hline $\begin{array}{l}\text { Pennellia brachycarpa Beilstein \& Al- } \\
\text { Shehbaz }\end{array}$ & Halimolobeae & endemic (AR) & 1067 & 3 & EN \\
\hline Pennellia yalaensis Salariato \& Al-Shehbaz & Halimolobeae & endemic (AR) & $4 *$ & 1 & $\mathrm{CR}$ \\
\hline Petroravenia eseptata Al-Shehbaz & Halimolobeae & endemic (AR) & 12 & 2 & EN \\
\hline $\begin{array}{l}\text { Phlebolobium maclovianum (d’Urv.) O.E. } \\
\text { Schulz }\end{array}$ & Thelypodieae & endemic (AR) & 935 & 3 & $\mathrm{EN}$ \\
\hline Physaria crassistigma O'Kane \& Al-Shehbaz & Physarieae & endemic (AR) & 1806 & 5 & EN \\
\hline Physaria lateralis O'Kane \& Al-Shehbaz & Physarieae & endemic (AR) & 311510 & 54 & $\mathrm{LC}$ \\
\hline $\begin{array}{l}\text { Physaria mendocina (Phil.) O'Kane \& Al- } \\
\text { Shehbaz }\end{array}$ & Physarieae & native (AR, UR) & 1001869 & 80 & $\mathrm{LC}$ \\
\hline Physaria okanensis Al-Shehbaz \& Prina & Physarieae & endemic (AR) & 1111 & 3 & EN \\
\hline Physaria pygmaea O'Kane \& Al-Shehbaz & Physarieae & endemic (AR) & 48514 & 11 & $\mathrm{LC}$ \\
\hline $\begin{array}{l}\text { Physaria urbaniana (Muschl.) O’Kane \& } \\
\text { Al-Shehbaz }\end{array}$ & Physarieae & native (AR, BO) & 161498 & 35 & $\mathrm{LC}$ \\
\hline $\begin{array}{l}\text { Polypsecadium arnottianum (Gillies ex Hook. } \\
\text { \& Arn.) Al-Shehbaz }\end{array}$ & Thelypodieae & endemic (AR) & 173825 & 24 & $\mathrm{LC}$ \\
\hline $\begin{array}{l}\text { Polypsecadium gilliesii (Romanczuk) Al- } \\
\text { Shehbaz }\end{array}$ & Thelypodieae & endemic (AR) & 381895 & 52 & $\mathrm{LC}$ \\
\hline $\begin{array}{l}\text { Polypsecadium grandiflorum Romanczuk \& } \\
\text { Boelcke }\end{array}$ & Thelypodieae & endemic (AR) & 68131 & 13 & $\mathrm{LC}$ \\
\hline $\begin{array}{l}\text { Polypsecadium harmsianum (Muschl.) O.E. } \\
\text { Schulz }\end{array}$ & Thelypodieae & native $(\mathrm{AR}, \mathrm{BO})$ & 29672 & 14 & $\mathrm{LC}$ \\
\hline $\begin{array}{l}\text { Polypsecadium magellanicum (Juss. ex Pers.) } \\
\text { Al-Shehbaz }\end{array}$ & Thelypodieae & native $(\mathrm{AR}, \mathrm{CH})$ & 123043 & 14 & $\mathrm{LC}$ \\
\hline $\begin{array}{l}\text { Polypsecadium tucumanense (O.E. Schulz) } \\
\text { Al-Shehbaz }\end{array}$ & Thelypodieae & endemic (AR) & 915 & 4 & $\mathrm{EN}$ \\
\hline Rorippa austroamericana Mart.-Laborde & Cardamineae & $\begin{array}{c}\text { native }(\mathrm{AR}, \mathrm{CH} \\
\mathrm{PE})\end{array}$ & 375736 & 12 & $\mathrm{LC}$ \\
\hline Rorippa bonariensis (Poir.) Macloskie & Cardamineae & $\begin{array}{l}\text { native (AR, BR, } \\
\text { CH, PA, UR) }\end{array}$ & 1197680 & 51 & $\mathrm{LC}$ \\
\hline Rorippa burkartii (Mart.-Laborde) Al-Shehbaz & Cardamineae & native (AR, BR) & 67257 & 8 & $\mathrm{LC}$ \\
\hline Rorippa clandestina (Spreng.) J.F. Macbr. & Cardamineae & $\begin{array}{c}\text { native }(\mathrm{AR}, \mathrm{BO} \\
\mathrm{BR}, \mathrm{PA}, \mathrm{PE},+)\end{array}$ & $8^{*}$ & 2 & $\mathrm{EN}$ \\
\hline
\end{tabular}


Table 1. (Continuation).

\begin{tabular}{|c|c|c|c|c|c|}
\hline Species & Tribe & Status & $\begin{array}{l}\mathbf{E O O} \\
\left(\mathbf{k m}^{2}\right)\end{array}$ & $\begin{array}{l}\mathrm{N}^{\circ} \text { of locations } \\
\text { based in } \\
0.1^{\circ} \text { cells }\end{array}$ & $\begin{array}{l}\text { IUCN category } \\
\text { based in } \\
\text { B1ab(iii) criteria }\end{array}$ \\
\hline Rorippa coxii (F. Phil. ex Phil.) L.E. Navas & Cardamineae & $\begin{array}{c}\text { native }(\mathrm{AR}, \mathrm{BR}, \\
\mathrm{CH})\end{array}$ & 48672 & 12 & $\mathrm{LC}$ \\
\hline Rorippa hilariana (Walp.) Cabrera & Cardamineae & $\begin{array}{l}\text { native (AR, BO, } \\
\text { BR, PA, UR) }\end{array}$ & 1671216 & 28 & $\mathrm{LC}$ \\
\hline Rorippa mandonii (E. Fourn.) Mart.-Laborde & Cardamineae & $\begin{array}{c}\text { native }(\mathrm{AR}, \mathrm{BO} \\
\mathrm{CH}, \mathrm{PA}, \mathrm{PE},+)\end{array}$ & 141758 & 23 & $\mathrm{LC}$ \\
\hline Rorippa nana (Schltdl.) J.F. Macbr. & Cardamineae & $\begin{array}{l}\text { native }(\mathrm{AR}, \mathrm{BO} \\
\mathrm{PE},+)\end{array}$ & 44618 & 9 & $\mathrm{LC}$ \\
\hline Rorippa philippiana Macloskie & Cardamineae & native $(\mathrm{AR}, \mathrm{CH})$ & 662290 & 11 & $\mathrm{LC}$ \\
\hline Sarcodraba andina O.E. Schulz & Thelypodieae & endemic (AR) & 21409 & 6 & NT \\
\hline $\begin{array}{l}\text { Sarcodraba dusenii (O.E. Schulz) Al- } \\
\text { Shehbaz }\end{array}$ & Thelypodieae & native $(\mathrm{AR}, \mathrm{CH})$ & 496 & 3 & EN \\
\hline $\begin{array}{l}\text { Sarcodraba karraikensis (Speg.) Gilg \& } \\
\text { Muschl. }\end{array}$ & Thelypodieae & endemic (AR) & 42531 & 7 & $\mathrm{LC}$ \\
\hline Sarcodraba subterranea O.E. Schulz & Thelypodieae & endemic (AR) & $4 *$ & 1 & $\mathrm{CR}$ \\
\hline Schizopetalon rupestre (Barnéoud) Reiche & Schizopetaleae & native $(\mathrm{AR}, \mathrm{CH})$ & 44109 & 13 & $\mathrm{LC}$ \\
\hline $\begin{array}{l}\text { Sibara mendocina (Boelcke \& S.C. Arroyo) } \\
\text { Al-Shehbaz }\end{array}$ & Thelypodieae & endemic (AR) & $4^{*}$ & 1 & CR \\
\hline Sibara tehuelches (Speg.) Al-Shehbaz & Thelypodieae & endemic (AR) & 68384 & 8 & $\mathrm{LC}$ \\
\hline Stenodraba chillanensis (Phil.) O.E. Schulz & Eudemeae & native $(\mathrm{AR}, \mathrm{CH})$ & 1464 & 7 & VU \\
\hline $\begin{array}{l}\text { Stenodraba colchaguensis (Barnéoud) O.E. } \\
\text { Schulz }\end{array}$ & Eudemeae & native $(\mathrm{AR}, \mathrm{CH})$ & 116468 & 22 & $\mathrm{LC}$ \\
\hline $\begin{array}{l}\text { Stenodraba imbricatifolia (Barnéoud) O.E. } \\
\text { Schulz }\end{array}$ & Eudemeae & native $(\mathrm{AR}, \mathrm{CH})$ & 8251 & 7 & VU \\
\hline Stenodraba lechleri (E. Fourn.) Ravenna & Eudemeae & native $(\mathrm{AR}, \mathrm{CH})$ & 1573 & 5 & EN \\
\hline Stenodraba parvifolia (Phil.) O.E. Schulz & Eudemeae & native $(\mathrm{AR}, \mathrm{CH})$ & 20849 & 14 & $\mathrm{LC}$ \\
\hline $\begin{array}{l}\text { Tomostima australis (R. Br.) Al-Shehbaz, M. } \\
\text { Koch \& Jordon-Thaden }\end{array}$ & Arabideae & $\begin{array}{c}\text { native (AR, } \mathrm{CH} \\
\mathrm{UR} \text { ) }\end{array}$ & 1328195 & 39 & $\mathrm{LC}$ \\
\hline $\begin{array}{l}\text { Trichotolinum deserticola (Speg.) O.E. } \\
\text { Schulz }\end{array}$ & Descuraineae & endemic (AR) & $8^{*}$ & 2 & EN \\
\hline Weberbauera densifolia Al-Shehbaz & Thelypodieae & native (AR, BO) & 14641 & 3 & VU \\
\hline $\begin{array}{l}\text { Weberbauera herzogii (O.E. Schulz) Al- } \\
\text { Shehbaz }\end{array}$ & Thelypodieae & $\begin{array}{c}\text { native }(\mathrm{AR}, \mathrm{BO} \\
\mathrm{PE})\end{array}$ & 6601 & 6 & VU \\
\hline Weberbauera orophila (A. Gray) O.E. Schulz & Thelypodieae & $\begin{array}{l}\text { native }(\mathrm{AR}, \mathrm{BO} \\
\mathrm{CH}, \mathrm{PE})\end{array}$ & 93673 & 15 & $\mathrm{LC}$ \\
\hline Weberbauera peruviana (DC.) Al-Shehbaz & Thelypodieae & $\begin{array}{l}\text { native }(\mathrm{AR}, \mathrm{BO} \\
\mathrm{CH}, \mathrm{PE})\end{array}$ & 21990 & 9 & NT \\
\hline Xerodraba colobanthoides Skottsb. & Eudemeae & endemic (AR) & 18594 & 5 & VU \\
\hline Xerodraba glebaria (Speg.) Skottsb. & Eudemeae & endemic (AR) & 807 & 3 & EN \\
\hline Xerodraba lycopodioides (Speg.) Skottsb. & Eudemeae & native $(\mathrm{AR}, \mathrm{CH})$ & 99484 & 17 & $\mathrm{LC}$ \\
\hline $\begin{array}{l}\text { Xerodraba monantha (Gilg ex Kuntze) } \\
\text { Skottsb. }\end{array}$ & Eudemeae & endemic (AR) & $4^{*}$ & 1 & CR \\
\hline $\begin{array}{l}\text { Xerodraba patagonica (Speg.) Skottsb. } \\
\text { patagonica }\end{array}$ & Eudemeae & native $(\mathrm{AR}, \mathrm{CH})$ & 143824 & 16 & $\mathrm{LC}$ \\
\hline $\begin{array}{l}\text { Xerodraba patagonica (Speg.) Skottsb. } \\
\text { pycnophylloides (Speg.) Salariato \& Al- } \\
\text { Shehbaz }\end{array}$ & Eudemeae & endemic (AR) & 74621 & 8 & $\mathrm{LC}$ \\
\hline $\begin{array}{l}\text { Yunkia subscandens (Kuntze) Salariato \& } \\
\text { Al-Shehbaz }\end{array}$ & Cremolobeae & native $(\mathrm{AR}, \mathrm{BO})$ & $8^{*}$ & 2 & EN \\
\hline $\begin{array}{l}\text { Zuloagocardamum jujuyensis Salariato \& } \\
\text { Al-Shehbaz }\end{array}$ & Thelypodieae & endemic (AR) & $4^{*}$ & 1 & $\mathrm{CR}$ \\
\hline
\end{tabular}


Overall, the province Jujuy included the greater numbers of species, threat categories, and Argentinean endemics (Table S1, Supplementary Material), followed by the provinces of Salta, Mendoza, Chubut, Santa Cruz, and Tucumán. A high number of endemics were also found in La Rioja and Catamarca. Regarding the species distribution along the Argentinean ecoregions, the Patagonian steppe and the Central Andean Puna were the regions with the highest number of species, threat categories, endemics, and even threatened endemics (Fig. 3, Table S2). Other ecoregions associated with different Andean environments, such as the Southern Andean steppe, the High Monte, and the Southern
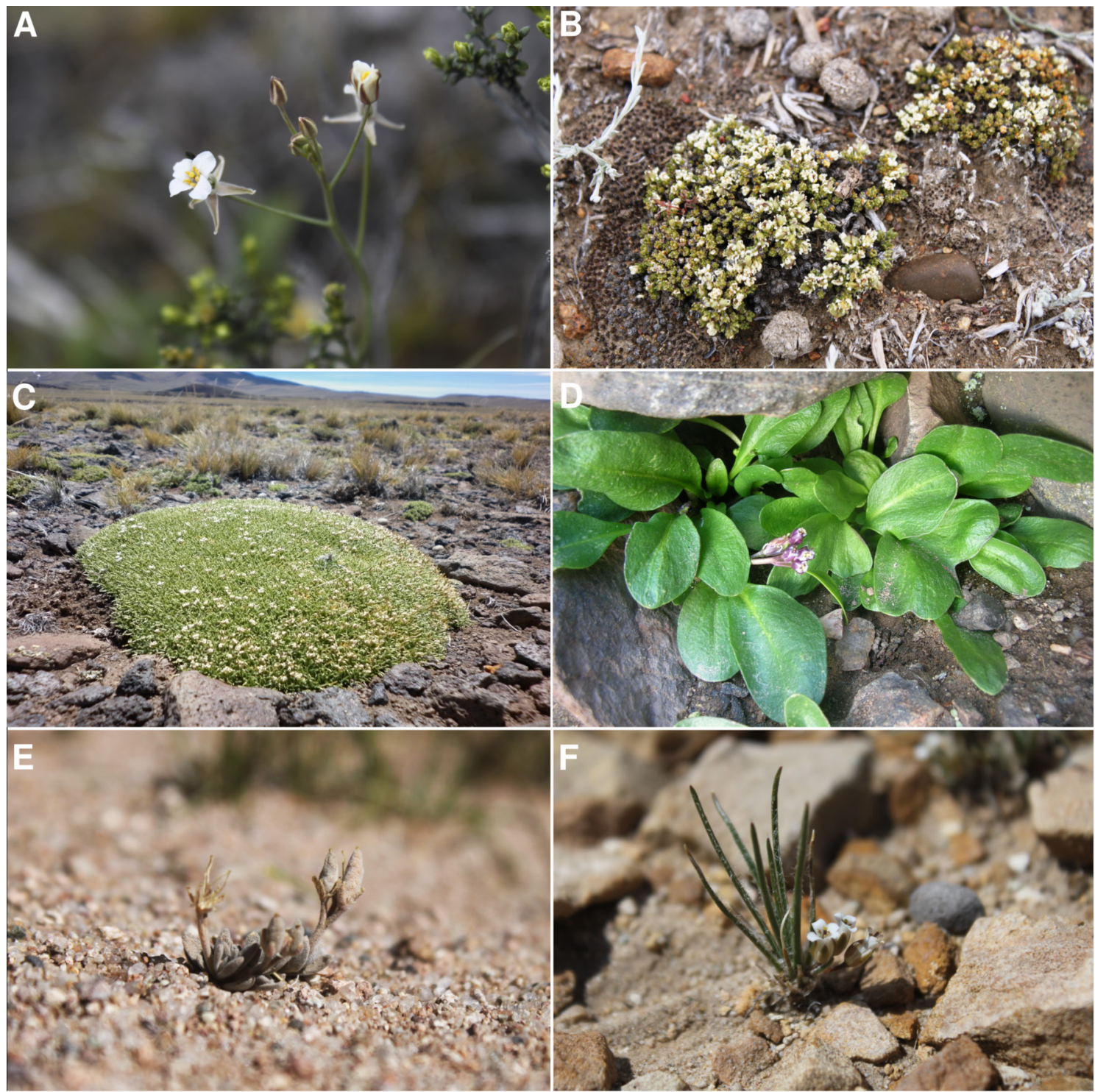

Fig. 2. Some endemic genera of Argentina. A, Chilocardamun patagonicum. B, Delpinophytum patagonicum. C, Lithodraba mendocinensis. D, Parodiodoxa chionophila. E, Petroravenia eseptata. F, Zuloagocardamum jujuyensis. Except for Chilocardamum, all other genera are monospecific. Photos by Fernando O. Zuloaga (A, F), Diego L. Salariato (B, C, E), and Soledad Cuello (D). Color version at http://www.ojs.darwin.edu.ar/index.php/ darwiniana/article/view/922/1199 
Andean Yungas, also showed high values for these variables (Fig. 3, Table S2). When we analyzed similarity of ecoregions in terms of their species composition using Jaccard distances we recovered three main clusters (Fig. 4): a cluster composed by the ecoregions associated with the Southern Andes (sensu Luebert \& Weigend, 2014)(Patagonian steppe, Magellanic Subpolar forest, Valdivian Temperate forest, and Southern Andean steppe ecoregions), a cluster including ecoregions associated with the central Andes (Central Andean Puna, High Monte, Southern Andean Yungas, and Central Andean Dry Puna ecoregions), and a cluster including ecoregions from central-eastern Argentina (Low Monte, Dry Chaco, Espinal, Humid Chaco, Paraná flooded savanna, and Humid Pampas ecoregions).

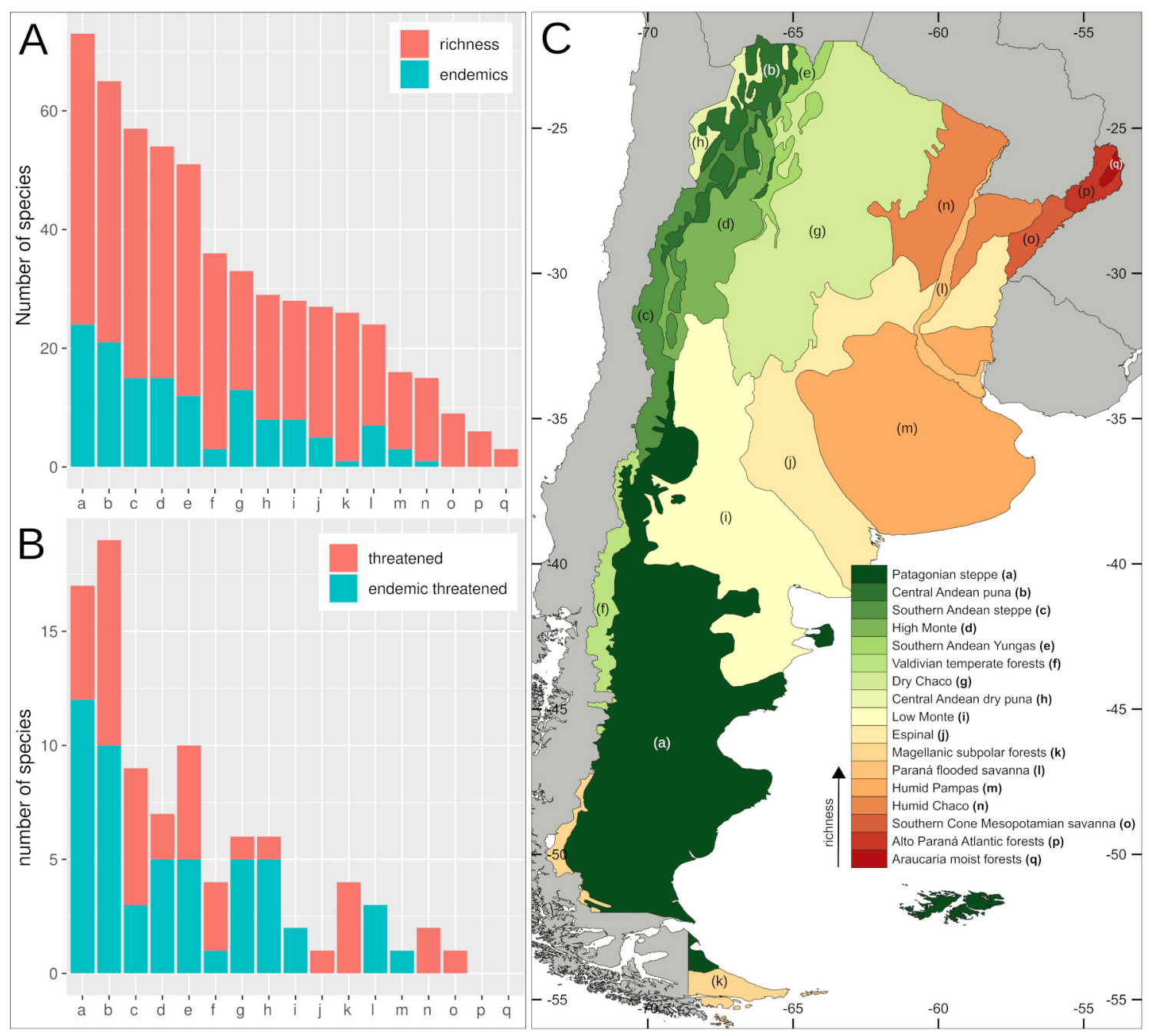

Fig. 3. Crucifer biodiversity throughout the ecoregions of Argentina. A, Barplot showing number of species (red) and endemics (blue) for the different ecoregions of Argentina. B, Number of threatened species (red) and threatened endemics (blue) for the different ecoregions of Argentina. C, Argentinean ecoregions colored according to their richness of crucifer species. Abbreviations: (a) Patagonian steppe, (b) Central Andean Puna, (c) Southern Andean steppe, (d) High Monte, (e) Southern Andean Yungas, (f) Valdivian Temperate forests, (g) Dry Chaco, (h) Central Andean Dry Puna, (i) Low Monte, (j) Espinal, (k) Magellanic Subpolar forests, (l) Paraná Flooded Savanna, (m) Humid Pampas, (n) Humid Chaco, (o) Southern Cone Mesopotamian Savanna, (p) Alto Paraná Atlantic forests, (q) Araucaria Moist forests. Color version at http://www.ojs.darwin.edu.ar/index.php/darwiniana/article/view/922/1199 
Some species that characterize ecoregions of the Southern Andes and Patagonia belong to Alshebazia Salariato \& Zuloaga, Cardamine L., Chilocardamum, Delpinophytum, Draba L., Litodraba, Menonvillea DC., Onuris Phil., Sarcodraba Gilg \& Muschl., Sibara Greene, Stenodraba O. E. Schulz, Trichotolinum, and Xerodraba Skottsb. Alternatively, species of Central Andean ecoregions mainly belong to Alshehbazia, Aschersoniodoxa Gilg \& Muschl., Brayopsis Gilg \& Muschl., Cremolobus DC., Dictyophragmus O. E. Schulz, Mancoa Wedd., Menonvillea, Neuontobotrys O. E. Schulz, Mostacillastrum O. E. Schulz, Parodiodoxa, Pennellia Nieuwl., Polypsecadium O. E. Schulz, Physaria (Nutt. ex
Torr. \& A. Gray) A. Gray, Weberbauera Gilg \& Muschl., and Zuloagocardamum. Ecoregions from central-eastern Argentina were mainly characterized by species of Cardamine, Descurainia Webb \& Berthel., Exhalimolobos Al-Shehbaz \& C. D. Bailey, Lepidium L., Mostacillastrum, Physaria, and Rorippa Scop.; while humid ecoregions of northeastern Argentina were mainly occupied by species of Cardamine, Lepidium, and Rorippa. Regarding the species distribution across biomes, the "montane grasslands and shrublands" biome (Table S3) exhibited the greater numbers of species, followed by the "temperate grasslands, savannas and shrublands" biome, the latter including the greater number of Argentinean endemics.

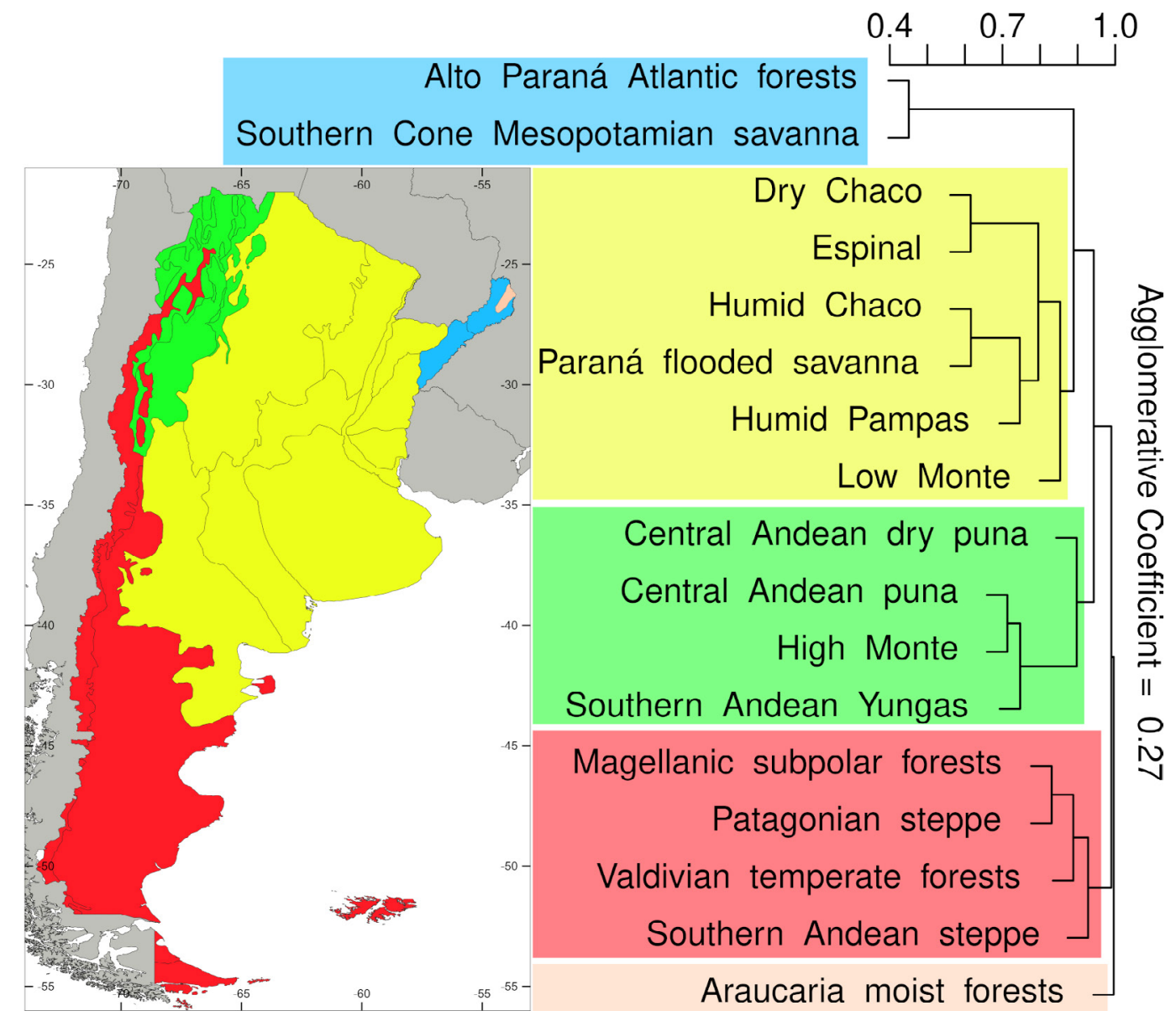

Fig. 4. Agglomerative hierarchical clustering for Argentine ecoregions using the UPGMA method and Jaccard distances based in their species composition (presence-absence data). The colored boxes denote the different clusters obtained, which are represented in the map on the right. Color version at http://www.ojs.darwin.edu.ar/index.php/darwiniana/ article/view/922/1199 
Spatial analyses using grids of 1 arc-degrees showed that diversity of the Argentinean Brassicaceae is concentrated along elevations of the Andes, the Precordillera, the internal mountain ranges (e.g., the "Sierras Pampeanas" and "Sierras transpampeanas" mountain systems), and lowlands of the Patagonian steppe (Fig. 5A). Species richness at tribal level showed that, except for tribes Cardamineae and Lepidieae, their distribution was mainly associated with the Andean regions and the Patagonian steppe (Figs. S2-S5, Supplementary Material).
Species richness was higher in cells located in: (c1) Jujuy province, Cochinoca and Humahuaca departments $\left(\sim 23.1^{\circ} \mathrm{S}, 65.8^{\circ} \mathrm{W}\right)$ along the Central Andean Puna ecoregion, and mainly associated with the highlands of "Sierra del Aguilar" and other secondary localities, such as "Tres Cruces, "Esquinas Blancas", and "Humahuaca" (Figs. 5A, S6 from Supplementary Material); (c2) Tucumán province, Tafi del Valle department $\left(\sim 26.5^{\circ} \mathrm{S}\right.$, $\left.65.8^{\circ} \mathrm{W}\right)$, primarily along the Central Andean Puna ecoregion and associated to the highlands
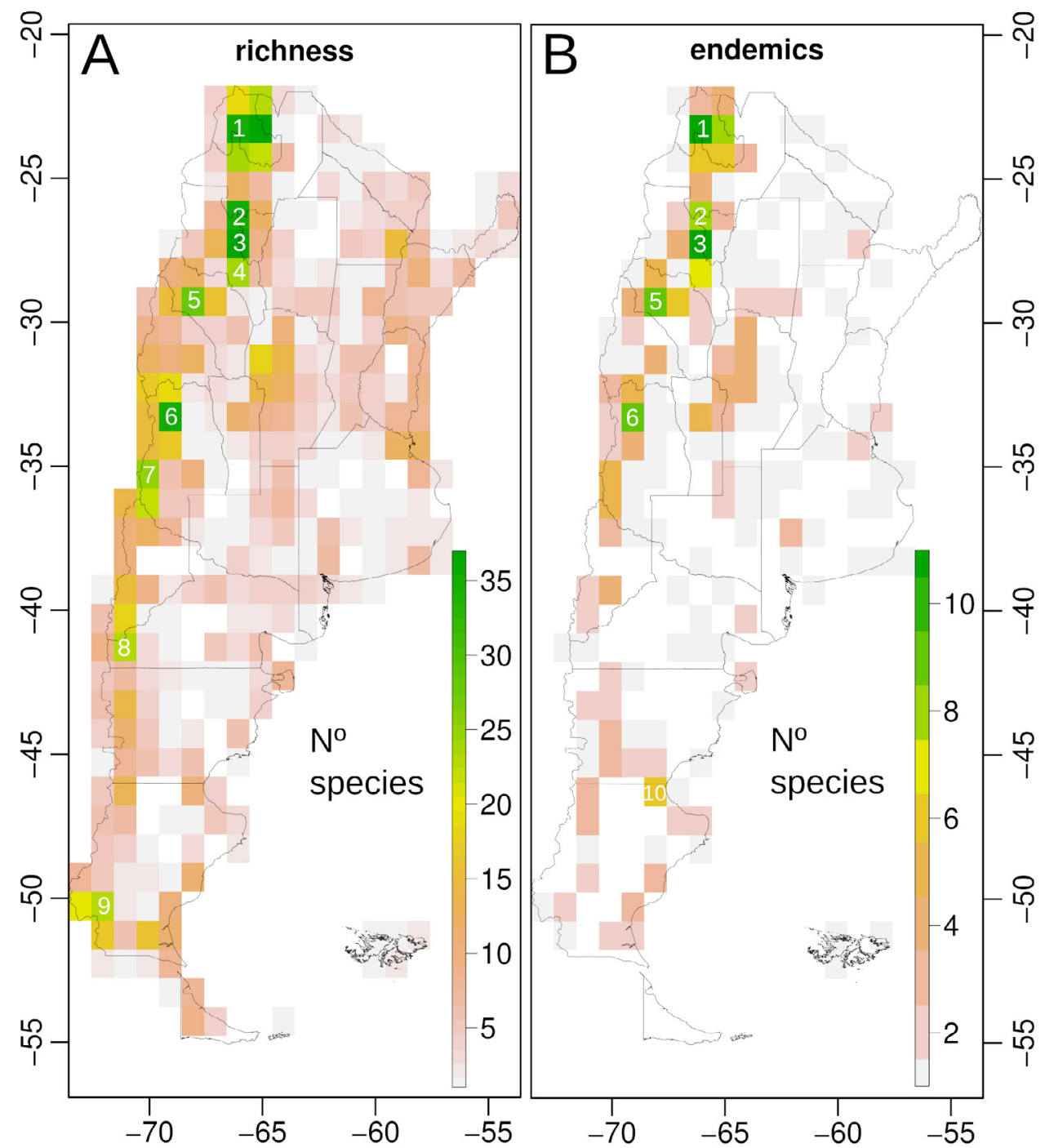

Fig. 5. Spatial patterns of biodiversity for Brassicaceae family in Argentina based on a spatial grid of 1 arc-degrees. A, Number of species (richness). B, Number of endemics (endemism). Cells with numbers are discussed in the text (see Results and Discussion sections). Color version at http://www.ojs.darwin.edu.ar/index.php/darwiniana/article/view/922/1199 
of the "Cumbres Calchaquíes" mountain system (e.g., "Cerro Negrito"); (c3) Tucumán (depts. Tafi del Valle and Chicligasta) and Catamarca (dept. Andalgalá) provinces $\left(\sim 27.0^{\circ} \mathrm{S}, 65.8^{\circ} \mathrm{W}\right)$, along the Central Andean Puna, Southern Andean Yungas, and High Monte ecoregions, and related to elevations of the "Sierra del Aconquija" mountain system (e.g. "Cerro el Bolsón", "Cerro Yutuyaco"); (c4) Catamarca province, Ambato department $\left(\sim 28.2 \mathrm{~S}^{\circ}, 66.0 \mathrm{~W}^{\circ}\right)$, in the High Monte and Dry Chaco ecoregions, and mainly associated with the highlands of the "Sierra del Manchao"; (c5) La Rioja province, Famatina department $\left(\sim 29.0^{\circ} \mathrm{S}, 67.8^{\circ} \mathrm{W}\right)$, within the High Monte ecoregion and associated with the mountain slopes of the "Sierra de Famatina"; (c6) north of the Mendoza province, Las Heras, Luján de Cuyo, Tupungato, and Tunuyán departments $(\sim 33.6 \mathrm{~S}$, $69.5 \mathrm{~W}$ ), along the Southern Andean steppe ecoregion, and mainly associated with the Andean highlands in "Cordón del Plata" (e.g., "Cerro del Plata") and "Portillo de Piuquenes"; (c7) south
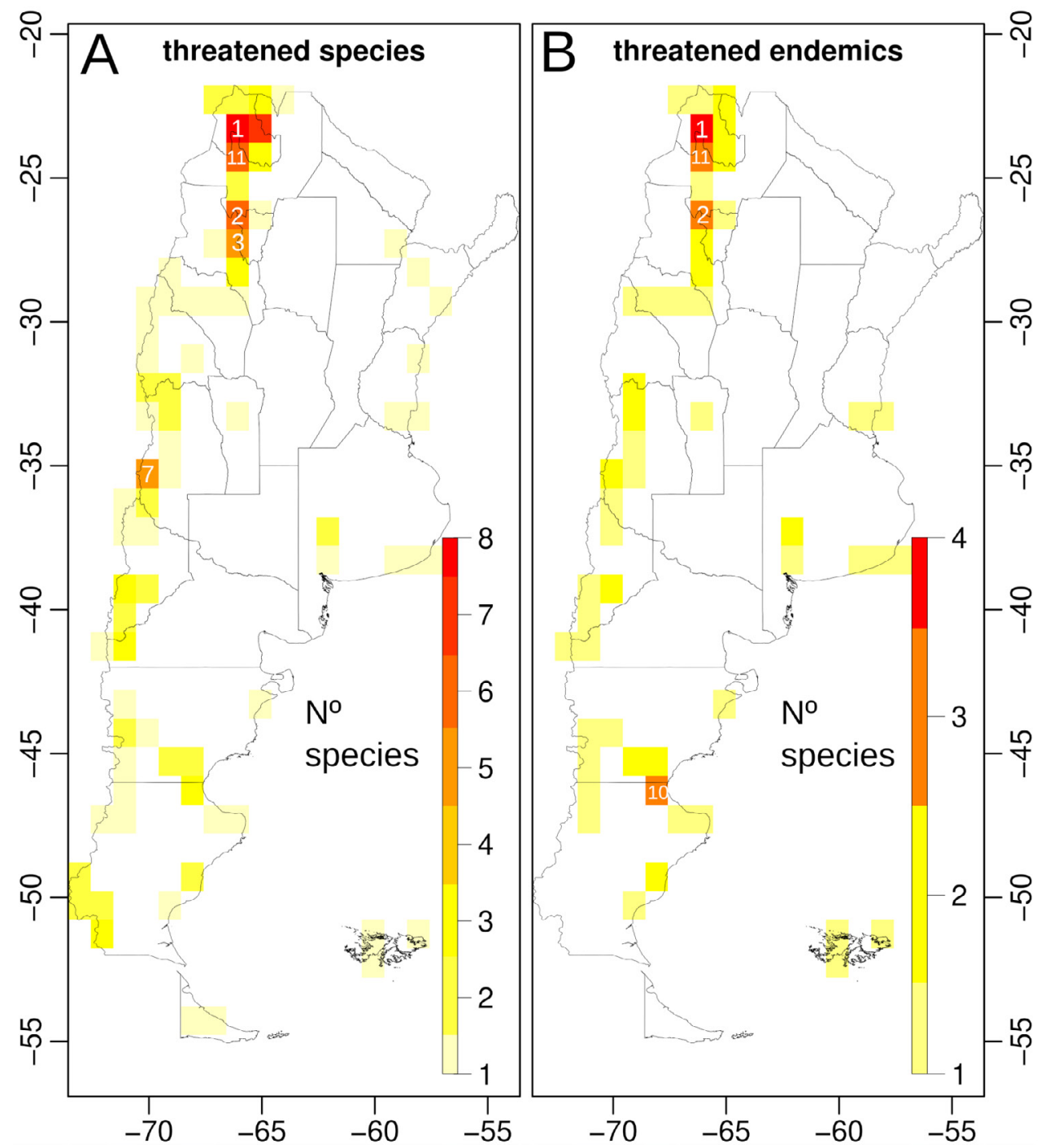

Fig. 6. Spatial patterns of threat for Brassicaceae family in Argentina based on a spatial grid of 1 arc-degrees. A, Number of threatened species. B, Number of threatened endemic species. Cells with numbers are discussed in the text (see Results and Discussion sections). Color version at http://www.ojs.darwin.edu.ar/index.php/darwiniana/article/view/922/1199 
of Mendoza province, Malargüe department $\left(\sim 35.1 \mathrm{~S}^{\circ}, 70.2 \mathrm{~W}^{\circ}\right)$, along the Southern Andean steppe ecoregion, and related to the Andean slopes along "Valle Hermoso" and the highlands of "Cerro Campanario" and "Paso Pehuenches"; (c8) Río Negro (dept. Bariloche) and Neuquén (dept. Los Lagos) provinces $\left(\sim 41.0 \mathrm{~S}^{\circ}, 71.4 \mathrm{~W}^{\circ}\right)$, along Valdivian Temperate forest and Patagonian steppe ecoregions, associated with the mountain slopes in the "Nahuel Huapi" national park (e.g., "Cerro Catedral", "Cerro Negro", "Cerro López", "Cerro Ventana", and "Cerro Nireco"); (c9) South of Santa Cruz province, Lago Argentino and Güer Aike departments $\left(\sim 50.5 \mathrm{~S}^{\circ}, 72.5 \mathrm{~W}^{\circ}\right)$, along the Magellanic Subpolar forest and Patagonian steppe ecoregions, associated with elevations in the "Los Glaciares" National Park (e.g., "Cerro Buenos Aires" and "Cordon de los Cristales"), "El Calafate" (e.g., "Cerro Calafate" and "Cerro Huyliches"), and the "Vizcachas" river basin (e.g., "Cerro de la Virgen", "Cerro Tridente", "Cerro Pináculo", and "Cerro Pan de Azúcar") (Fig. 5A). Endemic species were more represented in cells from "Sierra del Aguilar" and "Humahuaca" (c1), "Cumbres Calchaquíes" (c2), "Sierra del Aconquija" (c3), "Sierra de Famatina" (c5), "Cordón del Plata" (c6), and a new cell located in the east of the Chubut (dept. Escalante) - Santa Cruz (dept. Deseado) provinces (45.9S $\mathrm{S}^{\circ}$, $\left.68.4^{\circ} \mathrm{W}\right)(\mathrm{c} 10)$, along the Patagonian steppe and primarily associated with the "Pampa del Castillo" locality (Fig. 5B).
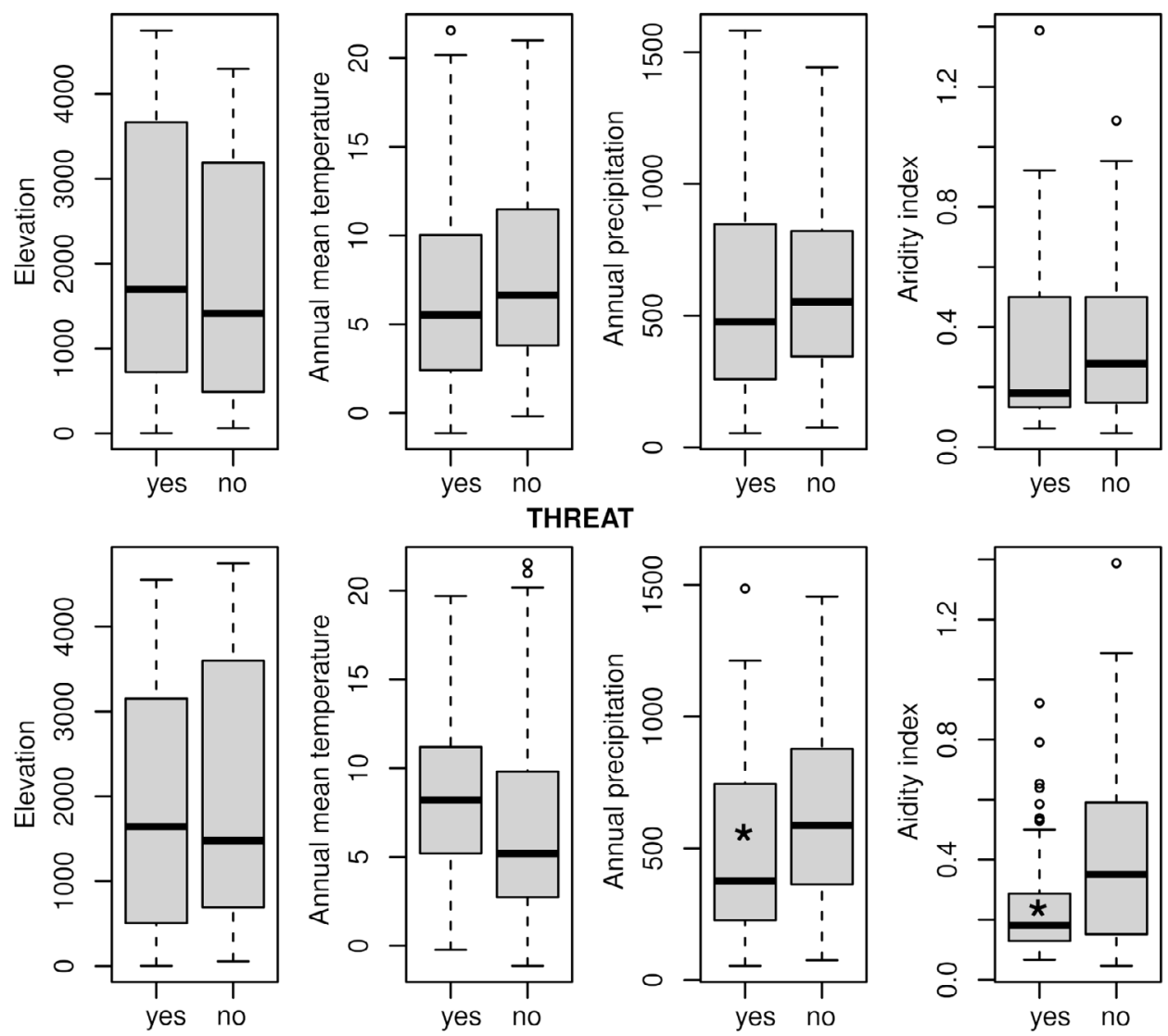

Fig. 7. Box-and-whisker plots showing values for elevation, annual mean temperature (BIO1), annual precipitation (BIO12) and aridity index (AI) between threatened vs. non-threatened species (above) and endemic vs. non-endemic species (below). Median (dark line), first and third quartile (boxes), and 95\% confidence interval of median (whiskers). Boxes denoted by an asterisk indicate $\mathrm{p} \leq 0.01$ for the Mann-Whitney $\mathrm{U}$ test. 
When analyzing the number of threatened species, cells from the Central Andean Puna of Jujuy ("Sierra del Aguilar" and "Humahuaca") (c1) plus highlands along "Cerro Tuzgle", "Abra Chorrillos" (Susques department) and "San Antonio de los Cobres" in Salta Province (Los Andes department) $\left(\sim 24.2^{\circ} \mathrm{S}, \quad 66.5^{\circ} \mathrm{W}\right)$ (c11); "Cumbres Calchaquies" (c2), "Sierra del Aconquija" (c3), and "Valle Hermoso" (c7), exhibited the highest values. (Fig. 6A). Alternatively, when examining number of threatened endemics, cells of "Sierra del Aguilar" (c1), "San Antonio de los Cobres" (c11), "Cumbres Calchaquíes" (c2), and "Pampa del Castillo" (c10) presented the highest values (Fig. 6A). Overall, cells from "Sierra del Aguilar" (c1) and "Cumbres Calchaquíes" (c2) showed the highest values for all variables. In addition, the cells with the least coverage of protected areas (including national, provincial and biosphere reserves) were those corresponding to the localities of "Sierra de Famatina" in La Rioja (c5) , "Valle Hermoso" and "Paso Pehuenches" in southern Mendoza (c7); the "Vizcachas" river basin in Southern Santa Cruz (c9), and "Pampa del Castillo" (c10) in southeastern Chubut (Fig. S7, Supplementary Material).

Spatial patterns using cells of 0.5 arcdegrees recovered a greater richness for the same localities as the one-degree cells, but at a finer scale (Fig. S8A, Supplementary Material). Threatened species were concentrated in "Sierra del Aguilar" (c1), "Cumbres Calchaquíes" (c2), and "Sierra del Aconquija" (c3) (Fig. S9A, Supplementary Material), while endemic species were mostly recovered in these localities together with "Sierra de Famatina" (c6), and "Cordón del Plata" (c7) (Fig. S8B). Threatened endemics were concentrated in "Sierra del Aguilar" (c1) and "Cumbres Calchaquíes" (c2) (Fig. S9B).

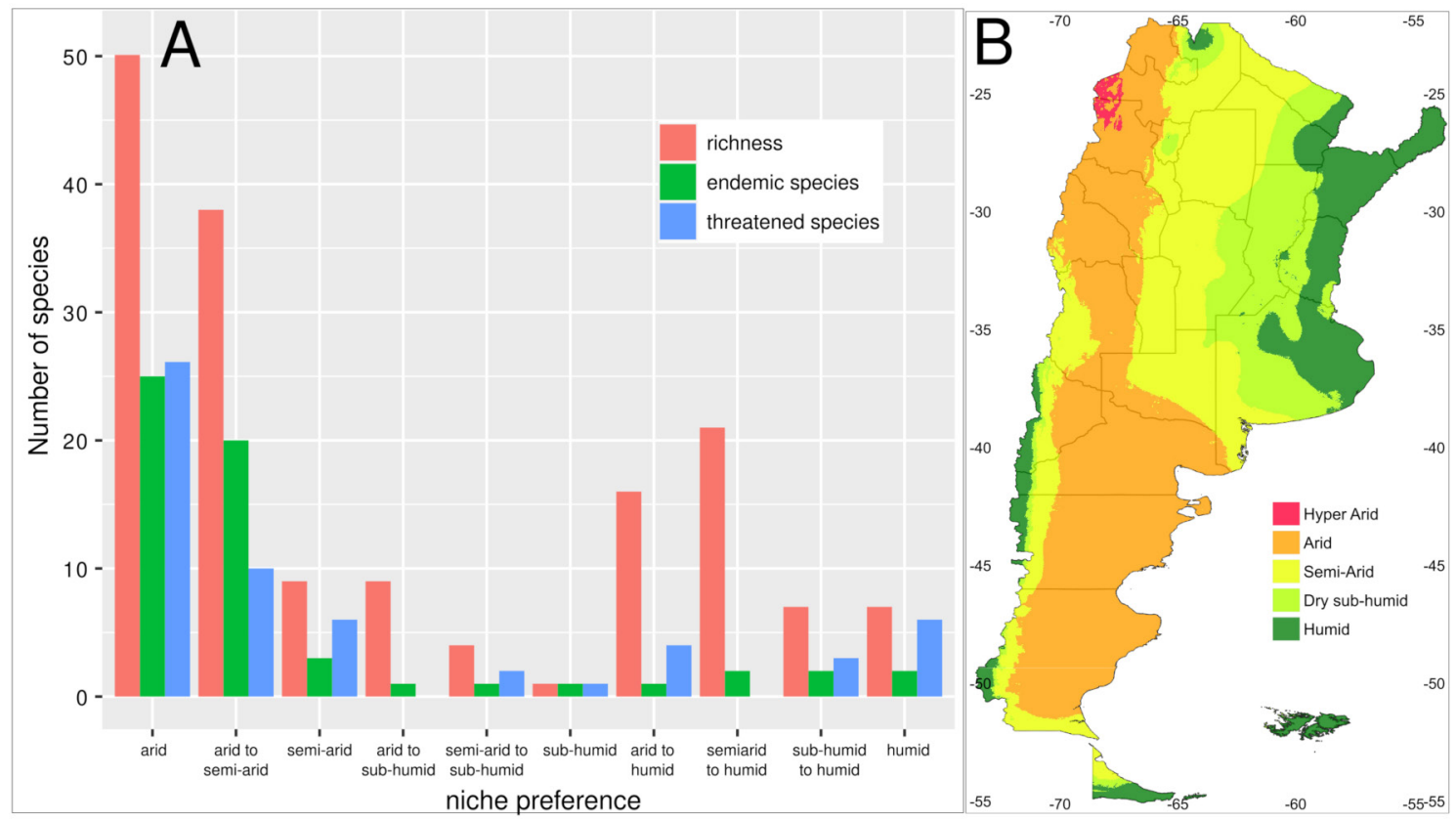

Fig. 8. Niche preference associated with aridity levels for different species of Argentinean crucifers. A, Bar plots showing number of species (red), number of endemics (green), and number of threatened species (blue) that inhabit different aridity regimes. Environments where species grow were categorized following the climate classification scheme for Aridity Index values (UNEP 1997) (<0.03: hyper Arid, 0.03 - 0.2: arid, 0.2 - 0.5: semi-arid. 0.5 - 0.65: dry sub-humid, $>0.65$ humid). For each species we used the AI values corresponding to the 5 th and 95 th percentile to capture the niche breadth. B, Geographic distribution of aridity categories in Argentina. Color version at http://www.ojs. darwin.edu.ar/index.php/darwiniana/article/view/922/1199 
Analysis using specimen occurrence values for elevation, annual mean temperature (BIO1), annual precipitation (BIO12), and aridity (AI) showed that threatened and non-threatened species were not differentiated for any of these variables (elevation: $\mathrm{p}=0.271$, BIO1: $\mathrm{p}=0.118$, BIO12: $p=0.584$, AI: $p=0.126$ ) (Fig. 7). Endemic and non-endemic species also did not show significant differences in elevation $(\mathrm{p}=0.207)$ and annual mean temperature $(\mathrm{p}=0.058)$, but they were differentiated for annual precipitation $(\mathrm{p}=0.004)$ and aridity $(\mathrm{p}<0.001)$, the endemic species showing lower mean annual precipitation and aridity (higher aridity) than native nonendemic species (Fig. 7). Finally, environment classification using the AI values (Fig. 8) showed that 97 species (out of 162 spp./subspp. present in Argentina; 60\%), 42 threatened species (out of $58 ; 72 \%$ ), and 48 endemic species (out of $58 ; 83 \%$ ) occupy exclusively arid to semi-arid environments. Furthermore, and following this classification, all localities identified in the spatial analyses (see above, Fig. 5A) corresponded to arid-semi arid environments, except those included in the "Nahuel Huapi" and "Los Glaciares" national parks (cells C8 and c9, respectively) (Fig. 8).

\section{DISCUSSION}

Crucifers are well represented in South America by ca. 406 native species (ca. 10\% of the family). These species inhabit a variety of different habitats along the biogeographical provinces of the North Andean Paramo, Puna, Prepuna, Altoandina, Yungas, and Subandean Patagonia (Cabrera \& Willink, 1980; Morrone, 2018). These regions, together with the Atacama-Sechura Desert, the Chilean Matorral, and the Patagonian steppe, provide a high diversity of habitats for the diversification of numerous plant groups (Luebert \& Weigend, 2014), including several lineages of this family (e.g., Toro-Núñez et al., 2013; Salariato et al., 2015b;2016;2018; 2020c). Events that occurred during the Neogene (e.g., Mid Miocene climatic optimum, uplift of the Andes, marine ingressions into the continent, changes in the Amazonian drainage system) and later during the
Pleistocene (e.g., climatic oscillations, glacialinterglacial cycles, aridification, changes in the sea level and seashores) had enormous effects on the diversification of the local biodiversity (Hoorn et al., 2010; Antonelli \& Sanmartín, 2011; Rull, 2011; Hazzi et al., 2018).

Regarding the Southern Cone of South America (Argentina, southern Brazil, Chile, Paraguay, and Uruguay), Argentina has the greatest number of vascular plant species (Zuloaga et al., 2019). Within the Brassicaceae, ca. $40 \%$ of the South American crucifers (160 species and two subspecies) are present in Argentina. Of these, 58 species were categorized here as threatened (VU, $\mathrm{EN}$, or $\mathrm{CR})(36 \%)$, however, when considering only endemism, more than half of the endemic species $(57 \%, 33$ spp.) are threatened. Species inhabit all environments and biogeographical regions of the country, although areas associated with the Andes and the Patagonian steppe contain most of the species. Arid to semi-arid environments in ecoregions of the Central Andean Puna, the Southern Andean steppe, the High Monte, and the Patagonian steppe harbor most of endemic and threatened species. These regions, corresponding to the "montane grasslands and shrublands" and "temperate grasslands, savannas and shrublands" biomes, are mostly associated with the South American Arid Diagonal (SAAD), a band of drylands that stretch diagonally across South America in a north-west to south-east direction (Garleff et al., 1991; Abraham et al., 2000; 2020). While humid regions in the country such as the Southern Andean Yungas or the Paraná Atlantic forest present high richness for vascular plants, main areas of endemism for vascular plants are found in the arid and semi-arid habitats of the Andes and the Patagonian steppe, with little endemism in the species rich humid forests (Zuloaga et al., 1999; Aagesen et al., 2012; Elías \& Aagesen, 2019). Nevertheless, crucifer species were nearly absent in the unique hyper arid region of the country located in western Catamarca and Salta (the only species present was Neuontobotrys tarapacana), in contrast to the high number of endemic species that grow in the Atacama Desert of Chile (Al-Shehbaz, 2010; Salariato et al., 2013b; Toro-Núñez et al., 2013; 2015). 
In our analyses, Central Andean Puna in northwestern Argentina (NOA) showed the highest values of richness, endemism, and threatened species, matching hotspots for richness and endemism. Hotspots of endemic vascular plants were reported for the arid ecoregions of NOA (Zuloaga et al. 1999; Aagesen et al., 2012; Godoy-Bürki et al., 2014), even though it has been reported that global hotspots of species richness are not congruent with endemism and threat (Orme et al., 205). Spatial congruence between richness and endemism has been detected in the NOA at a local scale and with specific plant families (Godoy-Bürki et al., 2014), including Poaceae (Aagesen al., 2009), Cactaceae (Ortega-Baes et al., 2012) and Brassicaceae (this study). Collections from localities from the NOA (e.g., Sierra del Aguilar in Jujuy and Cumbres Calchaquíes in Tucumán) correspond mostly to the Puna (between approx. $3500-4500 \mathrm{~m}$ ) and the Altoandina (above $4500 \mathrm{~m}$ ) biogeographical provinces, characterized by the montane shrublands (in the Puna) and grasslands (in the Altoandina) (Cabrera \& Willink, 1980). Some of the native "non-endemic" threatened species in these regions are Alshehbazia friesii, Aschersoniodixa cachensis, Mostacillastrum dianthoides, Weberbauera densifolia, and Weberbauera herzogii. On the other hand, threatened endemics are Dictyophragmus punensis, Draba burkartiana, Lepidium jujuyanum, Pennellia brachycarpa, Pennellia yalaensis, Petroravenia eseptata, Physaria okanensis, Polypsecadium tucumanense, and Zuloagocardamum jujuyensis.

Mountains of Sierra de Famatina in La Rioja also exhibited high richness for crucifers, being this family the fifth in number of species after Asteraceae, Poaceae, Solanaceae, and Fabaceae (Barboza et al., 2016). Highlands of this area, included in the High Monte ecoregion and mainly associated with the Puna and Altoandina biogeographical provinces, encompassed many of the species found in the Central Andean Puna of Jujuy, Tucumán, and Catamarca, and also species distributed to the South such as Draba magellanica, Menonvillea scapigera subsp longipes, and Neuontobotrys frutescens. Highlands of Sierra de Famatina are one of the main diversity hotspots of Argentina, but also one of the most deficient areas in terms of conservation; the need to include protected areas to conserve its biodiversity has been reported previously (Godoy-Bürky et al., 2014; Barboza et al., 2016). In this region Menonvillea famatinensis is the only threatened endemic.

Another area recovered with a high number of species and Argentinean endemics corresponds to the Andean highlands in Northern Mendoza (e.g., Cordón del Plata). This region, located in the southern portion of the Southern Andean steppe ecoregion, includes species mainly from the Cuyan High Andean district (Cabrera, 1971) as Cardamine volckmannii, Menonvillea cuneata, M. scapigera, $M$. spathulata, Mostacillastrum leptocarpum, and Neuontobotrys robusta. Mountains in this region were identified as a center of endemism of the genus Senecio L. (Asteraceae) and a potential cradle for its speciation (Elías \& Aagesen, 2019). Alternatively, along lowlands and valleys of this region, species from the Low Monte and Dry Chaco ecoregions, or Monte and Chaqueña biogeographical provinces (Cabrera \& Willink, 1980), are also present, including Physaria lateralis, P. mendocina, Exhalimolobos pazense, and E. weddellii. Threatened endemics in this area are Lithodraba mendocinensis, Physaria crassistigma, and Sibara mendocina.

The southernmost highlands of the Andes also present a high diversity of crucifer species. These areas, included in the Altoandina biogeographical province (Cabrera \& Willink, 1980) extend from central Mendoza southward to northern Tierra del Fuego, where highaltitude environments are found as islands on the mountain peaks of the Andes (Cabrera \& Willink, 1980; Padró et al., 2020). Southwards, species are generally shared with the Chilean Andes, so the number of endemics is lower than in the Central Andes. Mountains at Southern Mendoza and Northern Neuquén exhibits high species richness, and threatened species in this region are Lithodraba mendocinensis, Menonvillea cicatricosa, Neuontobotrys mendocina, and Stenodraba chillanensis. Eastern mountains of the Nahuel Huapi National Park $\left(\sim 41^{\circ} \mathrm{S}\right.$ latitude) 
also harbour a high number of species and two threatened endemics: Menonvillea comberi and $M$. rigida. Since precipitation decreases eastward, eastern mountains usually have a greater richness associated with their greater aridity. Eastern peaks have a higher diversity of environments, from dry slopes to humid forest spots, allowing the growth of both xeric and montane species, whereas western slopes lack arid environments. Furthermore, the snow cover is thinner and less persistent on the eastern mountains, while the higher amount of persistent snow covering some western peaks reduces the available area for plants (Ferreyra et al., 1998; Speziale et al., 2010).

High richness was also recovered in mountains of southern Santa Cruz, especially those located in the Vizcachas river basin. In this region, the Altoandina biogeographical province begins around $500-1000 \mathrm{~m}$ a. s. 1., while in northern Patagonian Andes Altoandina begins above 2000 m a. s. 1. (Cabrera \& Willink, 1980). Threatened species in this region are Alshehbazia hauthalii, Onuris hatcheriana, and Sarcodraba dusenii.

Arid lowlands in the southern portion of the Patagonian steppe also harbors a high number of species, but unlike species of the southern Andean slopes, most Patagonian species are endemic, with the steppe including even more endemics than the Central Andean Puna in the NOA. The Patagonian steppe, which acquired their current extensions at the end of the Neogene (Iglesias et al., 2011), is characterized by its dry and temperate-cold climate, with strong winds, snow during the winter, and frost almost all year (Cabrera \& Willink, 1980). Threatened endemics are mainly distributed along southeastern Chubut and eastern Santa Cruz, being some of these areas identified of conservation priority (Chehébar et al., 2013). Among threatened endemics are Chilocardamum longistylum, C. onuridifolium, Delpinophytum patagonicum, Lepidium santacruzensis, Sarcodraba subterranea, Trichotolinum deserticola, Xerodraba colobanthoides, $X$. glebaria, and X. monantha.

Other extra-Andean ecoregions, i.e. the Low Monte, the Dry Chaco, the Espinal, or the Humid Pampas showed considerably lower richness and endemism for the family; nevertheless, these regions also harbor threatened endemic species.
For example, humid pampas in Entre Rios province includes Lepidium burkartii, while in Buenos Aires Lepidium hickenii and Mostacillastrum ventanense grow, the latter is distributed along the Ventania Mountain System. In the Comechingones biogeographical province in San Luis inhabits Mostacillastrum carolinense. This biogeographical province, located in the Dry Chaco ecoregion, is characterized by moderate-altitude grasslands located in the Dry Chaco ecoregion, which, due to present a high number of endemics, has its own biogeographical categorization (Martínez et al., 2017).

Knowledge about biodiversity at a global level remains incomplete either because there is still uncertainty about the identity of the species living on Earth and their phylogenetic relationships (the Linnean and the Darwinian shortfalls, respectively), and because geographical distributions and ecological niches of most species are poorly understood and contains many gaps (the Wallacean and Hutchinsonian shortfalls, respectively) (Whittaker et al., 2005; Bini et al., 2006; Oliveira et al., 2016). These shortfalls are scale dependent, both on spatial and temporal dimensions, revealing different patterns of diversity by varying the scale of analysis (Whittaker et al., 2005). Using herbarium data, richness can be biased for some localities because they were more intensively sampled (due for example, to easier access to high mountain areas) generating a pattern of spatially biased sampling effort (Oliveira et al., 2016). Some poorly explored areas, as the high peaks of the southernmost Andes or the southern portion of Patagonia, need greater sampling effort in future field trips. Alternatively, most of the Argentinean species were relatively recently reviewed in taxonomic revision and in the Argentinean Flora (Al-Shehbaz, 2012a). However, for some species complexes, [e.g. Cardamine, Descurainia, Lepidium, Physaria] the taxonomic status of some populations and species limits are still unclear (e.g., Salariato \& Zuloaga, unpublished). Molecular studies with species-delimitation analyses that update and review species' geographic distribution, coupled with their taxonomic status, are necessary to provide basic information for biogeographic, 
ecological, systematic, and conservation studies (Riddle \& Hafner, 1999; Agapow et al., 2004; Isaac et al., 2004; Dayrat, 2005; Sukumaran \& Knowles, 2017). Furthermore, molecular phylogenies including nearly all South American species are urgently needed in order to estimate useful metrics for conservation, as the evolutionary distinctiveness (ED) (Isaac et al., 2007; Tucker et al., 2012) and the evolutionary distinct and globally endangered (EDGE) (Isaac et al., 2007; Redding et al., 2010) indices for species, or the phylogenetic diversity (PD) indices (Swenson, 2014; Tucker et al. 2017) for different regions of Argentina.

Mountain ecosystems of Argentina, along with the Patagonian steppe, are important centers of regional diversity and endemism (Zuloaga et al. 1999). Arid and semi-arid environments in the Andean region are home to many endemic and threatened species (Zuloaga et al., 1999; Aagesen et al., 2012; Godoy-Bürky et al., 2014; Elías \& Aagesen, 2019). However, and despite the relevance of habitat quality for biodiversity and ecosystem service policies, mountain environments are poorly known, making it difficult to establish rational conservation priorities on political agendas. Global warming together with cattle grazing, erosion, industrial activities, mining, and contamination of water supplies, are major threats in the region (Dinerstein et al., 1995; Aagesen, 2000; Gonzales, 2009). Therefore, effective conservation strategies are urgently needed to adequately preserve the endemic flora. Global and regional classification of species using the IUCN Threatened Species Categories, together with the analysis of spatial patterns of biodiversity constitute the first step to propose conservation strategies (Nic Lughadha et al., 2018; Rejmánek, 2018; Humphreys et al., 2019; Stévart et al., 2019). Results presented here on spatial patterns and the threat status of the Argentinean crucifers seeks to complement the information published in the flora of Argentina (Al-Shehbaz, 2012a), and hopes to be a useful contribution for future analyses of plant biodiversity patterns in the Southern Cone, as well as for the development of conservation plans.

\section{ACKNOWLEDGMENTS}

This work was funded by ANPCyT (Agencia Nacional de Promoción Científica y Tecnológica) grant PICT-2016-0096, CONICET (Consejo Nacional de Investigaciones Científicas y Técnicas) grant PIP112-201301-00124CO, and the National Geographic Society grant \#9841-16, for which we are profoundly grateful. Our deep gratitude goes to Dr. Ihsan A. Al-Shehbaz for the critical review of this work and his valuable support, guidance, and suggestions in the study of South American Brassicaceae over the years. Special thanks to Alfredo Grau and Soledad Cuello for generously providing the images of Parodiodoxa chionophila, and to Lone Aagesen, Juan M. Acosta and all other people who helped in many of the fieldtrips. We especially appreciate the help of Fernando Biganzoli and two anonymous reviewers who provided useful suggestions to improve the early version of this paper. Botanical collections associated to this project were conducted under the permits APN $\mathrm{N}^{\circ} 1103$ and $\mathrm{N}^{\circ} 1546$.

\section{BIBLIOGRAPHY}

Aagesen, D. 2000. Crisis and conservation at the end of the world: sheep ranching in Argentine Patagonia. Environmental Conservation 27: 208-215. DOI: https:// doi.org/10.1017/S0376892900000229

Aagesen, L; C. A. Szumik, F. O. Zuloaga \& O. Morrone. 2009. Quantitative biogeography in the South America highlands-recognizing the Altoandina, Puna and Prepuna through the study of Poaceae. Cladistics 25: 295-310. DOI: https://doi.org/10.1111/j.10960031.2009.00248. $\mathrm{x}$

Aagesen, L.; M. J. Bena, S. Nomdedeu, A. Panizza, R. P. López \& F. O. Zuloaga. 2012. Areas of endemism in the southern central Andes. Darwiniana 50: 218-251.

Abraham, E. M.; K. Garleff, H. Liebricht, A. Regarías, F. Schäbitz, F. A. Squeo, H. Stingl, H. Veit \& C. Villagrán. 2000. Geomorphology and palaeoecology of the arid diagonal in southern South America. Zietschrift für Angewandte Geologie SH 1: 55-61.

Abraham, E. M.; M. D. Rodríguez, M. C. Rubio, B. GuidaJohnson, L. Gomez \& C. Rubio. 2020. Disentangling the concept of "South American Arid Diagonal". Journal of Arid Environments 175: 104089. DOI: https://doi. org/10.1016/j.jaridenv.2019.104089 
Agapow, P. M.; O. R. Bininda-Emonds, K. A. Crandall, J. L. Gittleman, G. M. Mace, J. C. Marshall \& A. Purvis. 2004. The impact of species concept on biodiversity studies. The Quarterly Review of Biology 79: 161-179. DOI: https://doi. org $/ 10.1086 / 383542$

Al-Shehbaz, I. A. 2008. Brassicaceae, in F. O. Zuloaga, O. Morrone \& M. J. Belgrano (eds.), Catálogo de las Plantas Vasculares del Cono Sur. Monographs in Systematic Botany from the Missouri Botanical Garden 107: 1663-1709.

Al-Shehbaz, I. A. 2010. A synopsis of the genus Sibara (Brassicaceae). Harvard Papers in Botany 15(1): 139-147. DOI: https://doi.org/10.3100/025.015.0107

Al-Shehbaz, I. A. 2012a. Brassicaceae, in M. A. Anton \& F. O. Zuloaga (eds.), Flora Argentina, vol. 8, pp. 1-270. Buenos Aires: Editorial Sigma.

Al-Shehbaz, I. A. 2012b. A generic and tribal synopsis of the Brassicaceae (Cruciferae). Taxon 61: 931-954. DOI: https:// doi.org/10.1002/tax.615002

Antonelli, A. \& I. Sanmartín. 2011. Why are there so many plant species in the Neotropics? Taxon 60(2): 403-414. DOI: https://doi.org/10.1126/science.1194585

Bachman, S. P.; E. M. Nic Lughadha \& M. C. Rivers. 2018. Quantifying progress toward a conservation assessment for all plants. Conservation Biology 32: 516-524. DOI: https:// doi.org/10.1111/cobi.13071

Barboza, G. E.; J. J. Cantero, F. E. Chiarini, J. Chiapella, S. Freire, C. O. Nuñez, V. Palchetti \& A. L. Espinar. 2016. Vascular plants of Sierra de Famatina (La Rioja, Argentina): an analysis of its biodiversity. Phytotaxa 248(1): 1-123. DOI: https://doi.org/10.11646/phytotaxa.248.1.1

Bennun, L.; E. C. Regan, J. Bird, J. W. van Bochove, V. Katariya, S. Livingstone, R. Mitchell, C. Savy, M. Starkey, H. Temple \& J. D. Pilgrim. 2018. The value of the IUCN Red List for business decision making. Conservation Letters 11(1): e12353. DOI: https://doi.org/10.1111/conl.12353

Bini, L. M.; J. A. F. Diniz-Filho, T. F. Rangel, R. P. Bastos \& M. P. Pinto. 2006. Challenging Wallacean and Linnean shortfalls: knowledge gradients and conservation planning in a biodiversity hotspot. Diversity and Distributions 12(5): 475482. DOI: https://doi.org/10.1111/j.1366-9516.2006.00286.x

Bivand, R. S.; E. Pebesma \& V. Gomez-Rubio. 2013. Applied spatial data analysis with $R$, Second edition. New York: Springer. https://asdar-book.org/

Boelcke, O. 1967. Cruciferae, in A. L. Cabrera (ed.), Flora de la Provincia de Buenos Aires. Colección Cientifica del Instituto Nacional de Tecnología Agropecuaria, vol. 4(3): 281-371.

Boelcke, O. \& M. C. Romanczuk. 1984a. Cruciferae, in M. N. Correa (ed.), Flora Patagónica. Colección Cientifica del Instituto Nacional de Tecnología Agropecuaria, vol. 8(4a): 373-544.
Boelcke, O. \& M. C. Romanczuk. 1984b. Brassicaceae, in A. T. Hunziker (ed.), Los géneros de Fanerógamas de la Argentina. Boletín de la Sociedad Argentina de Botánica 23 (1-4): 76-84.

Boelcke, O. 1987. Cruciferae, in N. S. Troncoso \& N. M. Bacigalupo (eds.), Flora Ilustrada de Entre Ríos. Colección Cientifica del Instituto Nacional de Tecnología Agropecuaria, vol. 6(3): 358-414.

Boelcke, O. \& J. B. Martínez-Laborde. 1994. Cruciferae, in R. Kiesling (ed.), Flora de San Juan vol. 1 pp. 205-244. Buenos Aires: Vázques Mazzini.

Brook, B. W.; N. S. Sodhi \& C. J. A. Bradshaw. 2008. Synergies among extinction drivers under global change. Trends in Ecology and Evolution 23: 453-460. DOI: https://doi. org/10.1016/j.tree.2008.03.011

Brooks, T. M.; R. A. Mittermeier, G.A.B. da Fonseca, J. Gerlach, M. Hoffmann, J. F. Lamoreux, C.G. Mittermeier, J. D. Pilgrim \& A. S. L. Rodrigues. 2006. Global biodiversity conservation priorities. Science 313: 58-61. DOI: https:// doi.org/10.1126/science.1127609

Brooks, T. M.; S. H. Butchart, N. A. Cox, M. Heath, C. HiltonTaylor, M. Hoffmann, N. Kingston, J. P. Rodríguez, S. N. Stuart \& J. Smart. 2015. Harnessing biodiversity and conservation knowledge products to track the Aichi Targets and Sustainable Development Goals. Biodiversity 16(2-3): 157-174. DOI: https://doi.org/10.1080/14888386.2015.1075903

Brown, A.; U. Martínez Ortiz, M. Acerbi \& J. F. Corcuera. 2006. La situación ambiental Argentina 2005. Buenos Aires: Fundación Vida Silvestre Argentina.

Cabrera, A. L. 1971. Fitogeografía de la República Argentina. Boletín de la Sociedad Argentina de Botánica 14(1-2): 1-42.

Cabrera, A. L. \& A. Willink. 1980. Biogeografía de América latina. Washington DC: Programa Regional de Desarrollo Científico y Tecnológico.

Ceballos, G.; P. R. Ehrlich, A. D. Barnosky, A. García, R. M. Pringle \& T. M. Palmer. 2015. Accelerated modern humaninduced species losses: Entering the sixth mass extinction. Science advances 1(5), e1400253. DOI: https://doi. org/10.1126/sciadv. 1400253

Chen, I. C.; J. K. Hill, R. Ohlemüller, D. B. Roy \& C. D. Thomas. 2011. Rapid range shifts of species associated with high levels of climate warming. Science 333: 1024-1026. DOI: https://doi.org/10.1126/science.1206432

Chehébar, C.; A. Novaro, G. Iglesias, S. Walker, M. Funes, M. Tammone \& K. Didier. 2013. Identificación de áreas de importancia para la biodiversidad en la estepa y el monte de Patagonia. Buenos Aires: ErreGé y Asociados imprenta.

Corlett, R.T. 2016. Plant diversity in a changing world: status, trends, and conservation needs. Plant Diversity 38: 10-16. DOI: https://doi.org/10.1016/j.pld.2016.01.001 
Cuesta, F.; P. Muriel, L. D. Llambí, S. Halloy, N. Aguirre, S. Beck, J. Carilla, R. I. Meneses, S. Cuello, A. Grau, L. E. Gámez, J. Irazábal, J. Jácome, R. Jaramillo, L. Ramárez, N. Samaniego, D. Súarez-Duque, N. Thompson, A. Tupayachi, P .Viñas, K. Yager, M. T. Becerra, H. Pauli \& W. D. Gosling. 2017. Latitudinal and altitudinal patterns of plant community diversity on mountain summits across the tropical Andes. Ecography 40: 1381-1394. DOI: https://doi. org/10.1111/ecog.02567

Dayrat, B. 2005. Towards integrative taxonomy. Biological Journal of the Linnean Society 85(3): 407-417. DOI: https:// doi.org/10.1111/j.1095-8312.2005.00503.x

Dauby, G. 2020. ConR: Computation of Parameters Used in Preliminary Assessment of Conservation Status. R package version 1.3.0. https://CRAN.R-project.org/package=ConR

Dauby, G.; T. Stévart, V. Droissart, A. Cosiaux, V. Deblauwe, M. Simo-Droissart, M. S. M. Sosef, P. P. Lowry II, G. E. Schatz, R. E. Gereau \& T. L. P. Couvreur. 2017. ConR: An $\mathrm{R}$ package to assist large-scale multispecies preliminary conservation assessments using distribution data. Ecology and evolution 7: 11292-11303. DOI: https://doi.org/10.1002/ ece3.3704

Dinerstein, E.; D. M. Olson, D. J. Graham, A. L. Webster, S. A. Primm, M. P Bookbinder \& G. Ledec. 1995. Una evaluación del estado de conservación de las eco-regiones terrestres de América Latina y el Caribe. Washington, DC: Banco Mundial.

Dirzo, R. \& P. H. Raven. 2003. Global state of biodiversity and loss. Annual review of Environment and Resources 28: 137-167. DOI: https://doi.org/10.1146/annurev. energy.28.050302.105532

Elías, G. D. V. \& L. Aagesen. 2019. Areas of endemism and recent speciation in the Southern Cone of South America, using Senecio (Asteraceae) as a proxy. Biological Journal of the Linnean Society 128: 70-82. https://doi.org/10.1093/ biolinnean/blz070

Ferreyra, M.; A. Cingolani, C. Ezcurra \& D. Bran. 1998. High-Andean vegetation and environmental gradients in northwestern Patagonia, Argentina. Journal of Vegetation Science 9: 307-316. DOI: https://doi.org/10.2307/3237095

Gärdenfors, U.; C. Hilton-Taylor, G. M. Mace \& J. P. Rodríguez. 2001. The application of IUCN Red List criteria at regional levels. Conservation Biology 15(5): 1206-1212. DOI: https://doi.org//10.1111/j.1523-1739.2001.00112.x

Garleff, K.; F. Schäbitz, H. Stingi \& H. Veit.1991. Jungquartäre Landschaftentwicklung und Klimageschichte beiderseits der Ariden Diagonale Südamerikas. Bamberger Geographische Schriften 11: 359-394.

Gaston, K. J. 1994. Rarity. Dordrecht: Springer.
Godoy-Bürki, A. C.; P. Ortega-Baes, J. M. Sajama \& L. Aagesen. 2014. Conservation priorities in the Southern Central Andes: mismatch between endemism and diversity hotspots in the regional flora. Biodiversity and Conservation 23(1): 81-107. DOI: https://doi.org/10.1007/s10531-013-0586-1

Gonzales, J. A. 2009. Climatic change and other anthropogenic activities are affecting environmental services on the Argentina Northwest (ANW). IOP Conference Series, Earth and Environmental Science 6: 302014. DOI: http://dx.doi. org/10.1088/1755-1307/6/30/302014

Grau, R. H.; I. N. Gasparri \& M. T. Aide. 2005. Agriculture expansion and deforestation in seasonally dry forests of north-west Argentina. Environmental Conservation 32: 140148. DOI: https://doi.org/10.1017/S0376892905002092

Halloy, S. R. \& A. F. Mark. 2003. Climate-change effects on alpine plant biodiversity: A New Zealand perspective on quantifying the threat. Arctic, Antarctic, and Alpine Research 35: 248-254. DOI: https://doi.org/10.1657/15230430(2003)035[0248:CEOAPB]2.0.CO,2

Hazzi, N. A.; J. S. Moreno, C. Ortiz-Movliav \& R. D. Palacio. 2018. Biogeographic regions and events of isolation and diversification of the endemic biota of the tropical Andes. Proceedings of the National Academy of Sciences of United States of America 115(31): 7985-7990. DOI: https://doi. org/10.1073/pnas.1803908115

Hijmans, R. J. 2020. raster: Geographic Data Analysis and Modeling. R package version 3.1-5. https://CRAN.Rproject.org/package=raster

Hoorn, C.; F. P. Wesselingh, T. Steege, H. Bermudez, M. A. Mora, A. Sevink, I. Sanmartín, A. Sanchez-Meseguer, C. L. Anderson, J. P. Figueiredo, C. Jaramillo, D. Riff, F. R. Negri, H. Hooghiemstra, J. Lundberg, T. Stadler, T. Särkinen \& A. Antonelli. 2010. Amazonia through time: Andean uplift, climate change, landscape evolution, and biodiversity. Science 330: 927-931. DOI: https://doi.org/10.1126/ science. 1194585

Humphreys, A. M.; R. Govaerts, S. Z. Ficinski, E. Nic Lughadha \& M. S. Vorontsova. 2019. Global dataset shows geography and life form predict modern plant extinction and rediscovery. Nature Ecology \& Evolution 3(7): 1043-1047. DOI: https://doi.org/10.1038/s41559-019-0906-2

Iglesias, A.; A. E. Artabe, \& E. M. Morel. 2011. The evolution of Patagonian climate and vegetation from the Mesozoic to the present. Biological Journal of the Linnean Society 103(2): 409-422. DOI: https://doi.org/10.1111/j.10958312.2011.01657.x

INTA. 2009. Informe Técnico Unificado PNECO 1643. Cobertura del suelo de la República Argentina 2006-2007 (LCCS-FAO). Buenos Aires: INTA. 
IPCC. 2014. Climate change 2014: synthesis report, In: R. Pachauri \& L. Meyer (Eds.), Contribution of Working Groups I, II and III to the Fifth Assessment Report of the Inter-governmental Panel on Climate Change. Geneva: IPCC.

Isaac, N. J.; J. Mallet \& G. M. Mace. 2004. Taxonomic inflation: its influence on macroecology and conservation. Trends in Ecology \& Evolution 19(9): 464-469. DOI: https://doi. org/10.1016/j.tree.2004.06.004

Isaac, N. J.; S. T. Turvey, B. Collen, C. Waterman \& J. E. Baillie. 2007. Mammals on the EDGE: conservation priorities based on threat and phylogeny. PloS one 2(3): e296. DOI: https:// doi.org/10.1371/journal.pone.0000296

IUCN Species Survival Commission. 2012. IUCN Red List categories and criteria, version 3.1. Gland: IUCN. https:// portals.iucn.org/library/sites/library/files/documents/RL-2001001.pdf

IUCN Standards and Petitions Committee. 2019. Guidelines for Using the IUCN Red List Categories and Criteria, version 14. Prepared by the Standards and Petitions Committee. http:// www.iucnredlist.org/documents/RedListGuidelines.pdf

Jezkova, T. \& J. J. Wiens. 2016. Rates of change in climatic niches in plant and animal populations are much slower than projected climate change. Proceedings of the Royal Society B: Biological Sciences 283(1843): 20162104. DOI: https:// doi.org/10.1098/rspb.2016.2104

Karger, D. N.; O. Conrad, J. Böhner, T. Kawohl, H. Kreft, R. W. Soria-Auza, N. E. Zimmermann \& M. Kessler. 2017a. Climatologies at high resolution for the earth's land surface areas. Scientific data 4: 170122. DOI: https://doi. org/10.1038/sdata.2017.122

Karger, D. N.; O. Conrad, J. Böhner, T. Kawohl, H. Kreft, R. W. Soria-Auza, N. E. Zimmermann, H. P. Linder \& M. Kessler. 2017b. Data from: Climatologies at high resolution for the earth's land surface areas. Dryad Digital Repository. DOI: https://doi.org/10.5061/dryad.kd1d4

Lean, G.; D. Hinrichsen \& A. Markham. 1990. Atlas of the environment. London: Arrow Books Ltd.

Linder, H. P. 2001. Plant diversity and endemism in sub-Saharan tropical Africa. Journal of Biogeography 28: 169-182. DOI: https://doi.org/10.1046/j.1365-2699.2001.00527.x

Luebert, F. \& M. Weigend. 2014. Phylogenetic insights into Andean plant diversification. Frontiers in Ecology and Evolution 2: 27. DOI: https://doi.org/10.3389/ fevo.2014.00027

Maechler, M.; P. Rousseeuw, A. Struyf, M. Hubert \& K. Hornik. 2019. cluster: Cluster Analysis Basics and Extensions. $\mathrm{R}$ package version 2.1.0. https://cran.r-project.org/web/ packages/cluster
Martínez, G. A.; M. D. Arana, A. J. Oggero \& E. S. Natale. 2017. Biogeographical relationships and new regionalisation of high-altitude grasslands and woodlands of the central Pampean ranges (Argentina), based on vascular plants and vertebrates. Australian Systematic Botany 29(6): 473-488. DOI: https://doi.org/10.1071/SB16046

Martínez-Laborde, J. B. 1999. Brassicaceae, in O. Zuloaga \& F. Morrone (eds.), Catálogo de las Plantas Vasculares de la Argentina. Monographs in Systematic Botany from the Missouri Botanical Garden 74: 388-420.

Morea, J. P. 2014. Situación actual de la gestión de las áreas protegidas de la Argentina. Problemáticas actuales y tendencias futuras. Revista Universitaria de Geografia 23(1): 57-75.

Morello, J.; S. D. Matteucci, A. F. Rodriguez \& M. E. Silva. 2018. Ecorregiones y complejos Ecosistémicos de Argentina, Segunda Edición. Buenos Aires: FADU-GEPAMA.

Morrone, J. J. 2018. Evolutionary biogeography of the Andean region, CRC Biogeography Series. Boca Raton: CRC Press.

Nanni, A. S.; M. P. Rodríguez, D. Rodríguez, M. N. Regueiro, M. E. Periago, S. Aguiar, S. Ballari, C. Blundo, E. Derlindati, Y. Di Blanco, A. Eljall, H. R. Grau, L. Herrera, A. Huertas Herrera, A. E. Izquierdo, Lescano J. N., L. Macchi, F. Mazzini, M. Milkovic, L. Montti, A. Paviolo, M. Pereyra, R. Quintana, V. Quiroga, D. Renison, M. Santos Beade, A. Schaaf \& N.I. Gasparri. 2020. Presiones sobre la conservación asociadas al uso de la tierra en las ecorregiones terrestres de la Argentina. Ecología Austral 30(2): 304-320. DOI: https://doi.org/10.25260/EA.20.30.2.0.1056

Newbold, T.; L. N. Hudson, S. L. L. Hill, S. Contu, I. Lysenko, R. A. Senior, L. Börger, D. J. Bennett, A. Choimes, B. Collen, J. Day, A. De Palma, S. Díaz, S. EcheverriaLondoño, M. J. Edgar, A. Feldman, M. Garon, M. L. K Harrison, T. Alhusseini, D. J. Ingram, Y. Itescu, J. Kattge, V. Kemp, L. Kirkpatrick, M. Kleyer, D. Laginha Pinto Correia, C. D. Martin, S. Meiri, M. Novosolov, Y. Pan, H. R. P. Phillips, D. R. Purves, A. Robinson, J. Simpson, S. L. Tuck, E. Weiher, H. J. White, R. M. Ewers, G. M. Mace, J. P. W. Scharlemann \& A. Purvis. 2015. Global effects of land use on local terrestrial biodiversity. Nature 520(7545): 45-50. DOI: https://doi.org/10.1038/nature14324

Nic Lughadha, E.; B. E. Walker, C. Canteiro, H. Chadburn, A. P. Davis, S. Hargreaves, E. J. Lucas, A. Schuiteman, E. Williams, S. P. Bachman, D. Baines, A. Barker, A. P. Budden, J. Carretero, J. J. Clarkson, A. Roberts \& M. C. Rivers. 2018. The use and misuse of herbarium specimens in evaluating plant extinction risks. Philosophical transactions of the Royal Society B 374(1763): 20170402. DOI: https:// doi.org/10.1098/rstb.2017.0402 
Nic Lughadha, E.; S. P. Bachman, T. C. C. Leão, F. Forest, J. M. Halley, J. Moat, C. Acedo, K. L. Bacon, R. F. A. Brewer, G. Gâteblé, S. C. Gonçalves, R. Govaerts, P. M. Hollingsworth, I. Krisai-Greilhuber, E. J. de Lirio, P. G. P. Moore, R. Negrão, J. M. Onana, L. R. Rajaovelona, H. Razanajatovo, P. B. Reich, S. L. Richards, M. C. Rivers, A. Cooper, J. Iganci, G. P. Lewis, E. C. Smidt, A. Antonelli, G. M. Mueller \& B. E. Walker. 2020. Extinction risk and threats to plants and fungi. Plants, People, Planet, 2: 389-408. DOI: https:// doi.org/10.1002/ppp3.10146

Oliveira, U.; A. Pereira Paglia, A. D. Brescovit, C. J. B. de Carvalho, D. Paiva Silva, D. T. Rezende, F. Sá Fortes Leite, J. A. Nogueira Batista, J. P. Peixoto Pena Barbosa, J. R. Stehmann, J. S. Ascher, M. Ferreira de Vasconcelos, P. Löwenberg-Neto, P. Guimaräes Dias, P. De Marco Jr, V. Gianluppi Ferro \& A. J. Santos. 2016. The strong influence of collection bias on biodiversity knowledge shortfalls of Brazilian terrestrial biodiversity. Diversity and Distributions 22: 1232-1244. DOI: https://doi.org/10.1111/ ddi. 12489

Olson, D. M.; E. Dinerstein, E. D. Wikramanayake, N. D. Burgess, G. V. N. Powell, E. C. Underwood, J. A D'amico, I. Itoua, H. E. Strand, J. C. Morrison, C. J. Loucks, T. F. Allnutt, T. H. Ricketts, Y. Kura, J. F. Lamoreux, W. W. Wettengel, P. Hedao \& K. R. Kassem. 2001. Terrestrial ecoregions of the world: A new map of life on Earth. BioScience 51: 933-938. DOI: https://doi.org/10.1641/00063568(2001)051[0933:TEOTWA]2.0.CO,2

Oksanen, J., F. G. Blanchet, M. Friendly, R. Kindt, P. Legendre, D. McGlinn, P. R. Minchin, R. B. O’Hara, G. L. Simpson, P. Solymos, M. H. H. Stevens, E. Szoecs \& H. Wagner. 2019. vegan: Community Ecology Package. R package version 2.5-6. https://CRAN.R-project.org/package=vegan

Ortega-Baes, P.; S. Bravo, J. Sajama, S. Sühring, J. Arrueta, E. Sotola, M. Alonso-Pedano, A. C. Godoy-Bürki, N. R. Frizza, G. Galíndez, P. Gorostiague, A. Barrionuevo \& A. Scopel. 2012. Intensive field surveys in conservation planning: Priorities for cactus diversity in the Saltenian Calchaquíes Valleys (Argentina). Journal of Arid Environments 82: 9197. DOI: https://doi.org/10.1016/j.jaridenv.2012.02.005

Padró, A.; V. Hechem \& J. J. Morrone. 2020. Biogeographic characterisation of the Austral High Andean district, Patagonian province, based on vascular plant taxa. Australian Systematic Botany 33: 174-190. DOI: https://doi. org/10.1071/SB19005

Paruelo, J. M.; J. P. Guerschman, G. Piñeiro, E. G. Jobbagy, S. R. Verón, G. Baldi \& S. Baeza. 2006. Cambios en el uso de la tierra en Argentina y Uruguay: marcos conceptuales para su análisis. Agrociencia 10(2): 47-61.
Pebesma, E. J. \& R.S. Bivand. 2005. Classes and methods for spatial data in R. R News 5(2): 9-13. https://cran.r-project. org/doc/Rnews/Rnews_2005-2.pdf

Prina, A. O. 1995a. Las Crucíferas de la Provincia de La Pampa, República Argentina. Revista de la Facultad de Agronomía de la Universidad Nacional de La Pampa 8: 29-67.

Prina, A. O. 1995b. Brassicaceae, Flora del Valle de Lerma. Aportes Botánicos de Salta, Serie Flora 3(2): 1-81.

Quantum GIS Development Team. 2016. QGIS Geographic Information System. Open Source Geospatial Foundation. http://qgis.org

R Core Team. 2020. $R$ : A language and environment for statistical computing. Vienna: R Foundation for Statistical Computing. https://www.R-project.org

Redding, D. W.; C. V. DeWolff \& A. Ø. Mooers. 2010. Evolutionary distinctiveness, threat status, and ecological oddity in primates. Conservation Biology 24(4): 1052-1058. DOI: https://doi.org/10.1111/j.1523-1739.2010.01532.x

Rejmánek, M. 2018. Vascular plant extinctions in California: A critical assessment. Diversity and Distributions 24(1): 129136. DOI: https://doi.org/10.1111/ddi.12665

Ricketts, T. H.; E. Dinerstein, T. Boucher, T. M. Brooks, S. H. M. Butchart, M. Hoffmann, J. F. Lamoreux, J. Morrison, M. Parr, J. D. Pilgrim, A. S. L. Rodrigues, W. Sechrest, G. E. Wallace, K. Berlin, J. Bielby, N. D. Burgess, D. R. Church, N. Cox, D. Knox, C. Loucks, G. W. Luck, L. L. Master, R. Moore, R. Naidoo, R. Ridgely, G. E. Schatz, G. Shire, H. Strand, W. Wettengel, \& E. Wikramanayake. 2005. Pinpointing and preventing imminent extinctions. Proceedings of the National Academy of Sciences 102(51): 18497-18501. DOI: https://doi.org/10.1073/ pnas.0509060102

Riddle, B. R. \& D. J. Hafner. 1999. Species as units of analysis in ecology and biogeography: time to take the blinders off. Global Ecology and Biogeography 8(6): 433-441. DOI: https://doi.org/10.1046/j.1365-2699.1999.00170.x

Rodrigues, A. S.; J. D. Pilgrim, J. F. Lamoreux, M. Hoffmann \& T. M. Brooks. 2006. The value of the IUCN Red List for conservation. Trends in Ecology \& Evolution 21(2): 71-76. DOI: https://doi.org/10.1016/j.tree.2005.10.010

Rull, V. 2011. Neotropical biodiversity: Timing and potential drivers. Trends in Ecology \& Evolution 26(10): 508-513. DOI: https://doi.org/10.1016/j.tree.2011.05.011

Salariato, D. L.; F. O. Zuloaga \& I. A. Al-Shehbaz. 2012. Morphometric studies and taxonomic delimitation in Menonvillea scapigera and related species (Cremolobeae: Brassicaceae). Plant Systematics and Evolution 298(10): 1961-1976. DOI: https://doi.org/10.1007/s00606-0120694-5 
Salariato, D. L.; F. O. Zuloaga \& I. A. Al-Shehbaz. 2013a. Revision and tribal placement of the Argentinean genus Parodiodoxa (Brassicaceae). Plant Systematics and Evolution 299(2): 305-316. DOI: https://doi.org/10.1007/ s00606-012-0722-5

Salariato, D. L.; F. O. Zuloaga \& I. A. Al-Shehbaz. 2013b. Molecular phylogeny of Menonvillea and recognition of the new genus Aimara (Brassicaceae: Cremolobeae). Taxon 62(6): 1220-1234. DOI: https://doi.org/10.12705/626.6

Salariato, D. L. \& I. A. Al-Shehbaz. 2014. Zuloagocardamum (Brassicaceae: Thelypodieae) a new genus from the Andes highlands of northern Argentina. Systematic Botany 39(2): 563-577. DOI: https://doi.org/10.1600/036364414X680898

Salariato, D. L.; F. O. Zuloaga \& I. A. Al-Shehbaz. 2014. A revision of the genus Menonvillea (Cremolobeae, Brassicaceae). Phytotaxa 162(5): 241-298. DOI: https://doi. org/10.1600/036364414X680898

Salariato, D. L. \& Zuloaga, F. O. 2015. Taxonomic placement of Onuris hauthalii (Brassicaceae: Eudemeae), based on morphology and multilocus species tree analyses, and the recognition of the new genus Alshehbazia. Kew Bulletin 70(4): 49. DOI: https://doi.org/10.1007/s12225-0159602-9

Salariato, D. L.; F. O. Zuloaga \& I. A. Al-Shehbaz. 2015a. A taxonomic revision of the genus Xerodraba (Eudemeae, Brassicaceae). Phytotaxa 207(1): 39-67. DOI: https://doi. org/10.11646/phytotaxa.207.1.2

Salariato, D. L.; F. O. Zuloaga, A. Cano, \& I. A. Al-Shehbaz. 2015b. Molecular phylogenetics of tribe Eudemeae (Brassicaceae) and implications for its morphology and distribution. Molecular Phylogenetics and Evolution 82: 4359. DOI: https://doi.org/10.1016/j.ympev.2014.09.030

Salariato, D. L.; F. O. Zuloaga, A. Franzke, K. Mummenhoff \& I. A. Al-Shehbaz. 2016. Diversification patterns in the CES clade (Brassicaceae tribes Cremolobeae, Eudemeae, Schizopetaleae) in Andean South America. Botanical Journal of the Linnean Society 181: 543-566. DOI: https:// doi.org/10.1111/boj.12430

Salariato, D. L.; I. A. Al-Shehbaz \& F. O. Zuloaga. 2018. Reinstatement of the southern Andean genus Stenodraba (Brassicaceae) based on molecular data and insights from its environmental and geographic distribution. Systematic Botany 43: 35-52. DOI: https://doi. org/10.1600/036364418X697085

Salariato, D. L.; F. O. Zuloaga \& I. A. Al-Shehbaz. 2019. Pennellia yalaensis (Brassicaceae: Halimolobeae), a new species from the Andes of northern Argentina. Systematic Botany 44(2): 355-362. DOI: https://doi.org/10.1600/03636 $4419 X 15562052252252$
Salariato, D. L.; F. O. Zuloaga \& I. A. Al-Shehbaz. 2020a. A reevaluation of the Andean Genus Petroravenia (Brassicaceae: Thelypodieae) based on morphological and molecular data. Journal of Systematics and Evolution 58(1): 43-58. DOI: https://doi.org/10.1111/jse.12486

Salariato, D. L.; H. Trinidad, A. Cano, F. O. Zuloaga \& I. A. Al-Shehbaz. 2020b. Molecular data reveal hidden diversity in the central Andean species Weberbauera spathulifolia (Thelypodieae: Brassicaceae). Botanical Journal of the Linnean Society 193: 523-545. DOI: https://doi.org/10.1093/ botlinnean/boaa026

Salariato, D. L.; A. Cano, F. O. Zuloaga \& I. A. Al-Shehbaz. 2020c. Molecular phylogeny of Cremolobus (Brassicaceae) supports the recognition of the new genus Yunkia and demonstrates the high habitat diversity of tribe Cremolobeae. Systematics and Biodiversity 18: 295-314. DOI: https://doi. org/10.1080/14772000.2020.1739777

Schatz, G. E. 2002. Taxonomy and herbaria in service of plant conservation: Lessons from Madagascar's endemic families. Annals of the Missouri Botanical Garden 89: 145-152. DOI: https://doi.org/10.2307/3298559

Speziale, K. L.; A. Ruggiero \& C. Ezcurra. 2010. Plant species richness-environment relationships across the SubantarcticPatagonian transition zone. Journal of Biogeography 37(3): 449-464. DOI: https://doi.org/10.1111/j.13652699.2009.02213.x

Stévart, T.; G. Dauby, P. P. Lowry, A. Blach-Overgaard, V. Droissart, D. J. Harris, B. A. Mackinder, G. E. Schatz, B. Sonké, M. S. M. Sosef, J. C. Svenning, J. J. Wieringa \& T. L. P. Couvreur. 2019. A third of the tropical African flora is potentially threatened with extinction. Science advances 5(11): eaax9444. DOI: https://doi.org/10.1126/sciadv. aax 9444

Sukumaran, J. \& L. L. Knowles. 2017. Multispecies coalescent delimits structure, not species. Proceedings of the National Academy of Sciences 114(7): 1607-1612. DOI: https://doi. org/10.1073/pnas.1607921114

Swenson, N. G. 2014. Functional and phylogenetic ecology in R. New York: Springer.

Thiers B. 2020. [continuously updated, accessed March 2020]. Index herbariorum: a global directory of public herbaria and associated staff. The Bronx: New York Botanical Garden's Virtual Herbarium. http://sweetgum.nybg.org/science/ih

Toro-Núñez, O.; M. E. Mort, E. Ruiz-Ponce \& I. A. AlShehbaz. 2013 Phylogenetic relationships of Mathewsia and Schizopetalon (Brassicaceae) inferred from nrDNA and cpDNA regions: taxonomic and evolutionary insights from an Atacama Desert endemic lineage. Taxon 62: 343-356. DOI: https://doi.org/10.12705/622.4 
Toro-Núñez, O.; I. A. Al-Shehbaz \& M. E. Mort. 2015. Phylogenetic study with nuclear and chloroplast data and ecological niche reveals Atacama (Brassicaceae), a new monotypic genus endemic from the Andes of the Atacama Desert, Chile. Plant Systematics and Evolution 301(5): 13771396. DOI: https://doi.org/10.1007/s00606-014-1157-y

Trabucco, A. \& R. Zomer. 2019. Global Aridity Index and Potential Evapotranspiration (ET0) Climate Database v2, accessed August 2020. figshare Dataset. DOI: https://doi. org/10.6084/m9.figshare.7504448.v3

Tucker, C. M.; M. W. Cadotte, T. J. Davies \& T. G. Rebelo. 2012. Incorporating geographical and evolutionary rarity into conservation prioritization. Conservation Biology 26(4): 593601. DOI: https://doi.org/10.1111/j.1523-1739.2012.01845.x

Tucker, C. M.; M. W. Cadotte, S. B. Carvalho, T. J. Davies, S. Ferrier, S. A. Fritz, R. Grenyer, M. R. Helmus, L. S. Jin, A. O. Mooers, S. Pavoine, O. Purschke, D. W. Redding, D. F. Rosauer, M. Winter \& F. Mazel. 2017. A guide to phylogenetic metrics for conservation, community ecology and macroecology. Biological Reviews 92(2): 698-715. DOI: https://doi.org/10.1111/brv.12252

United Nations Environment Programme (UNEP). 1997. World Atlas of Desertification, second edition. London: Oxford University Press.
Urban, M. C. 2015. Accelerating extinction risk from climate change. Science 348: 571-573. DOI: https://doi.org/10.1126/ science.aaa4984

Vallejos, M.; J. N. Volante, M. J. Mosciaro, L. M. Vale, M. L. Bustamante \& J. M. Paruelo. 2015. Transformation dynamics of the natural cover in the Dry Chaco ecoregion: a plot level geo-database from 1976 to 2012. Journal of Arid Environments 123: 3-11. DOI: https://doi.org/10.1126/science.aaa4984

Volante, J. N.; D. Alcaraz-Segura, M. J. Mosciaro, E. F. Viglizzo \& J. M. Paruelo. 2012. Ecosystem functional changes associated with land clearing in NW Argentina. Agriculture, Ecosystems \& Environment 154: 12-22. DOI: https://doi. org/10.1016/j.agee.2011.08.012

Whittaker R. J.; M. B. Araújo, P. Jepson, R. J. Ladle, J. M. E. Watson \& K. J. Willis. 2005. Conservation Biogeography: assessment and prospect. Diversity and Distributions 11: 3-23. DOI: https://doi.org/10.1111/j.1366-9516.2005.00143.x

Zuloaga, F. O.; O. Morrone \& D. Rodríguez. 1999. Análisis de la biodiversidad en plantas vasculares de la Argentina. Kurtziana 27(1): 17-167.

Zuloaga, F. O.; M. J. Belgrano \& C. A. Zanotti. 2019. Actualización del Catálogo de las Plantas Vasculares del Cono Sur. Darwiniana, nueva serie 7(2): 208-278. DOI: https://doi.org/10.14522/darwiniana.2019.72.861

\section{SUPPLEMENTARY MATERIAL}

Table S1. Number of species (richness), endemics, threatened species, and threatened endemics for political provinces of Argentina.

\begin{tabular}{|c|c|c|c|c|}
\hline \multirow[b]{2}{*}{ Province } & \multicolumn{4}{|c|}{ Number of species } \\
\hline & Richness & Endemics & Threatened & Threatened endemics \\
\hline Buenos Aires & 20 & 4 & 2 & 2 \\
\hline Catamarca & 43 & 13 & 6 & 3 \\
\hline Chaco & 13 & 1 & 1 & 0 \\
\hline Chubut & 47 & 14 & 8 & 6 \\
\hline Córdoba & 22 & 5 & 0 & 0 \\
\hline Corrientes & 15 & 2 & 1 & 0 \\
\hline Distrito Federal & 9 & 1 & 0 & 0 \\
\hline Entre Ríos & 16 & 2 & 2 & 1 \\
\hline Formosa & 9 & 1 & 0 & 0 \\
\hline Jujuy & 55 & 14 & 15 & 7 \\
\hline La Pampa & 16 & 3 & 0 & 0 \\
\hline La Rioja & 39 & 14 & 2 & 2 \\
\hline Mendoza & 48 & 11 & 7 & 4 \\
\hline Misiones & 8 & 0 & 0 & 0 \\
\hline Neuquén & 43 & 10 & 7 & 4 \\
\hline Río Negro & 35 & 6 & 3 & 1 \\
\hline Salta & 49 & 13 & 10 & 4 \\
\hline San Juan & 36 & 8 & 3 & 1 \\
\hline San Luis & 15 & 5 & 1 & 1 \\
\hline Santa Cruz & 46 & 12 & 10 & 6 \\
\hline Santa Fe & 14 & 3 & 0 & 0 \\
\hline Santiago del Estero & 14 & 3 & 0 & 0 \\
\hline Tierra del Fuego & 14 & 1 & 2 & 1 \\
\hline Tucumán & 45 & 12 & 8 & 4 \\
\hline
\end{tabular}


D. L. SALARIATO \& F. O. ZULOAGA. Diversity and conservation status of crucifers

Table S2. Number of species (richness), endemics, threatened species, and threatened endemics for ecoregions of Argentina (sensu Olson et al. 2001).

\begin{tabular}{lcccc} 
& \multicolumn{2}{c}{ Number of species } & \\
\cline { 2 - 4 } \multicolumn{1}{c}{ Ecoregion } & Richness & Endemics & Threatened & Threatened endemics \\
Alto Paraná Atlantic forests & 6 & 0 & 0 & 0 \\
Araucaria moist forests & 3 & 0 & 0 & 0 \\
Central Andean dry puna & 29 & 8 & 6 & 5 \\
Central Andean puna & 65 & 21 & 19 & 10 \\
Dry Chaco & 33 & 13 & 6 & 5 \\
Espinal & 27 & 5 & 1 & 0 \\
High Monte & 53 & 15 & 6 & 5 \\
Humid Chaco & 15 & 1 & 2 & 0 \\
Humid Pampas & 24 & 7 & 3 & 3 \\
Low Monte & 28 & 8 & 2 & 2 \\
Magellanic subpolar forests & 26 & 1 & 1 & 0 \\
Paraná flooded savanna & 16 & 3 & 17 & 1 \\
Patagonian steppe & 73 & 24 & 9 & 12 \\
Southern Andean steppe & 57 & 12 & 10 & 3 \\
Southern Andean Yungas & 51 & 15 & 1 & 5 \\
Southern Cone Mesopotamian savanna & 9 & 0 & 4 & 0 \\
Valdivian temperate forests & 36 & 3 & & 1
\end{tabular}

Table S3. Number of species (richness), endemics, threatened species, and threatened endemics for biomes of Argentina (sensu Olson et al. 2001).

\begin{tabular}{lcccc} 
& & \multicolumn{2}{c}{ Number of species } \\
\cline { 2 - 4 } \multicolumn{1}{c}{ Biome } & Richness & Endemics & Threatened & Threatened endemics \\
\hline Flooded grasslands and savannas & 18 & 3 & 2 & 1 \\
Montane grasslands and shrublands & 96 & 29 & 28 & 15 \\
Temperate broadleaf and mixed forests & 49 & 4 & 8 & 1 \\
Temperate grasslands, savannas and shrublands & 92 & 33 & 21 & 15 \\
Tropical and subtropical grasslands, savannas and shrublands & 36 & 13 & 8 & 5 \\
Tropical and subtropical moist broadleaf forests & 53 & 15 & 10 & 5
\end{tabular}

Table S4. Number of species (richness), endemics, threatened species, and threatened endemics for tribes present in Argentina.

\begin{tabular}{lcccc}
\multicolumn{1}{c}{ Tribe } & \multicolumn{2}{c}{ Number of species } \\
\hline Arabideae & Richness & Endemics & Threatened & Threatened endemics \\
Cardamineae & 10 & 2 & 2 & 1 \\
Coluteocarpeae & 21 & 0 & 3 & 0 \\
Cremolobeae & 1 & 0 & 0 & 0 \\
Descuraineae & 14 & 5 & 7 & 3 \\
Eudemeae & 8 & 1 & 1 & 1 \\
Halimomobeae & 23 & 5 & 12 & 4 \\
Lepidieae & 10 & 3 & 4 & 3 \\
Physarineae & 26 & 10 & 7 & 5 \\
Schizopetaleae & 6 & 4 & 2 & 2 \\
Thelypodieae & 1 & 0 & 0 & 0 \\
\hline
\end{tabular}




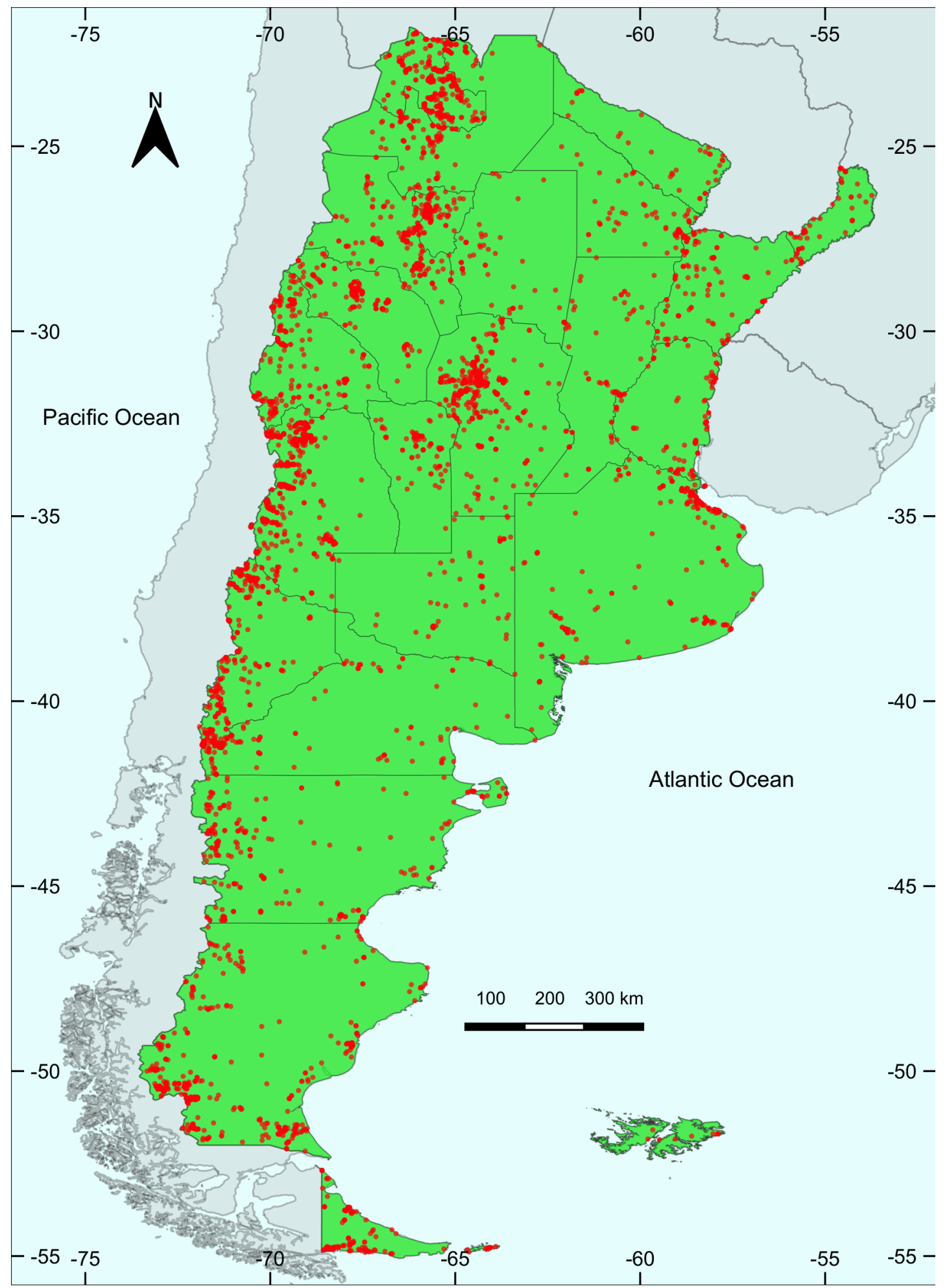

Fig. S1. Study area (Argentina) and occurrences for Brassicaceae species analyzed in this work. Color version at http:// www.ojs.darwin.edu.ar/index.php/darwiniana/article/view/922/1199 
D. L. SALARIATO \& F. O. ZULOAGA. Diversity and conservation status of crucifers
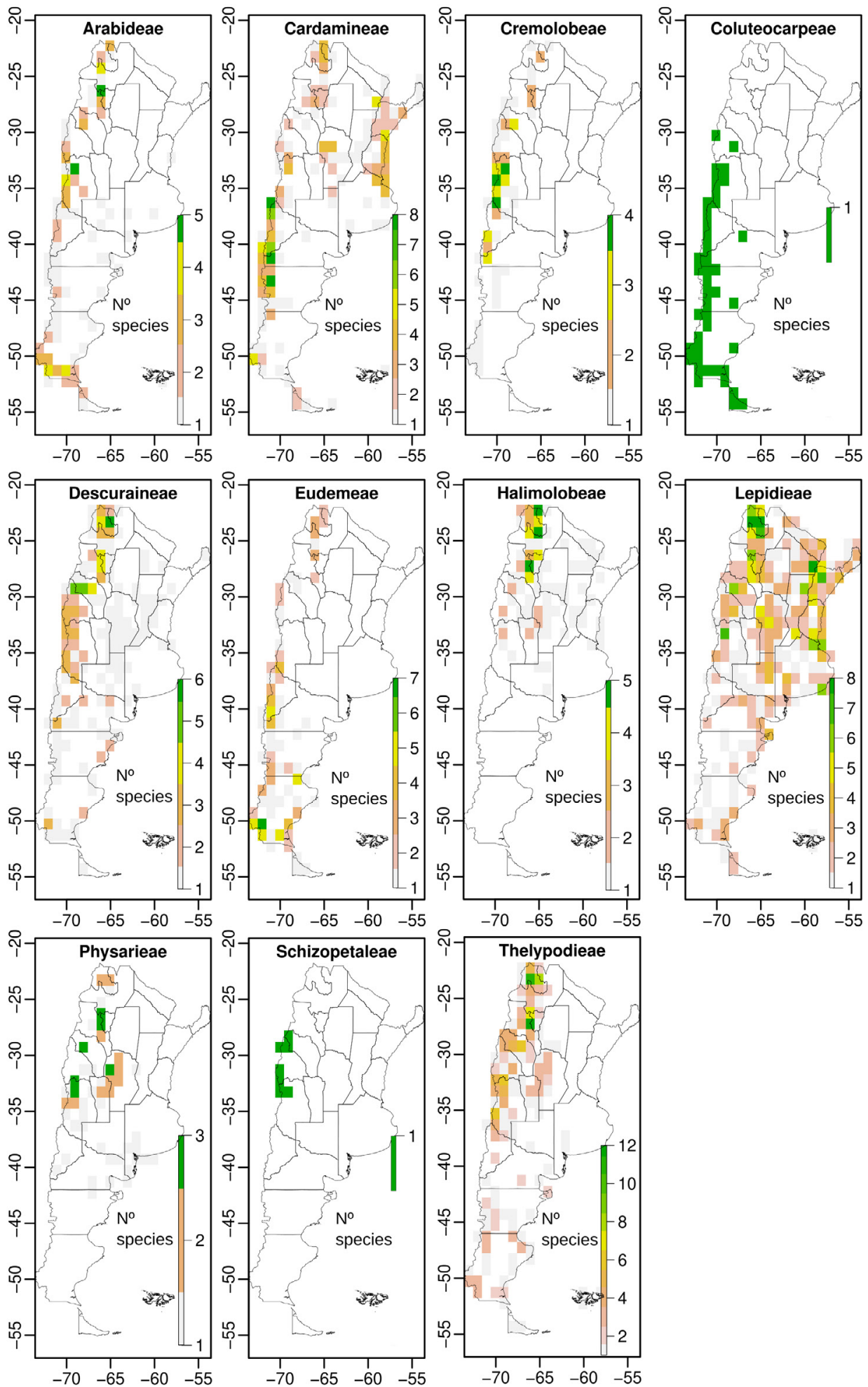

Fig. S2. Spatial patterns of richness (number of species) for tribes of Brassicaceae family in Argentina based on a spatial grid of 1 arc-degrees. Color version at http://www.ojs.darwin.edu.ar/index.php/darwiniana/article/view/922/1199 

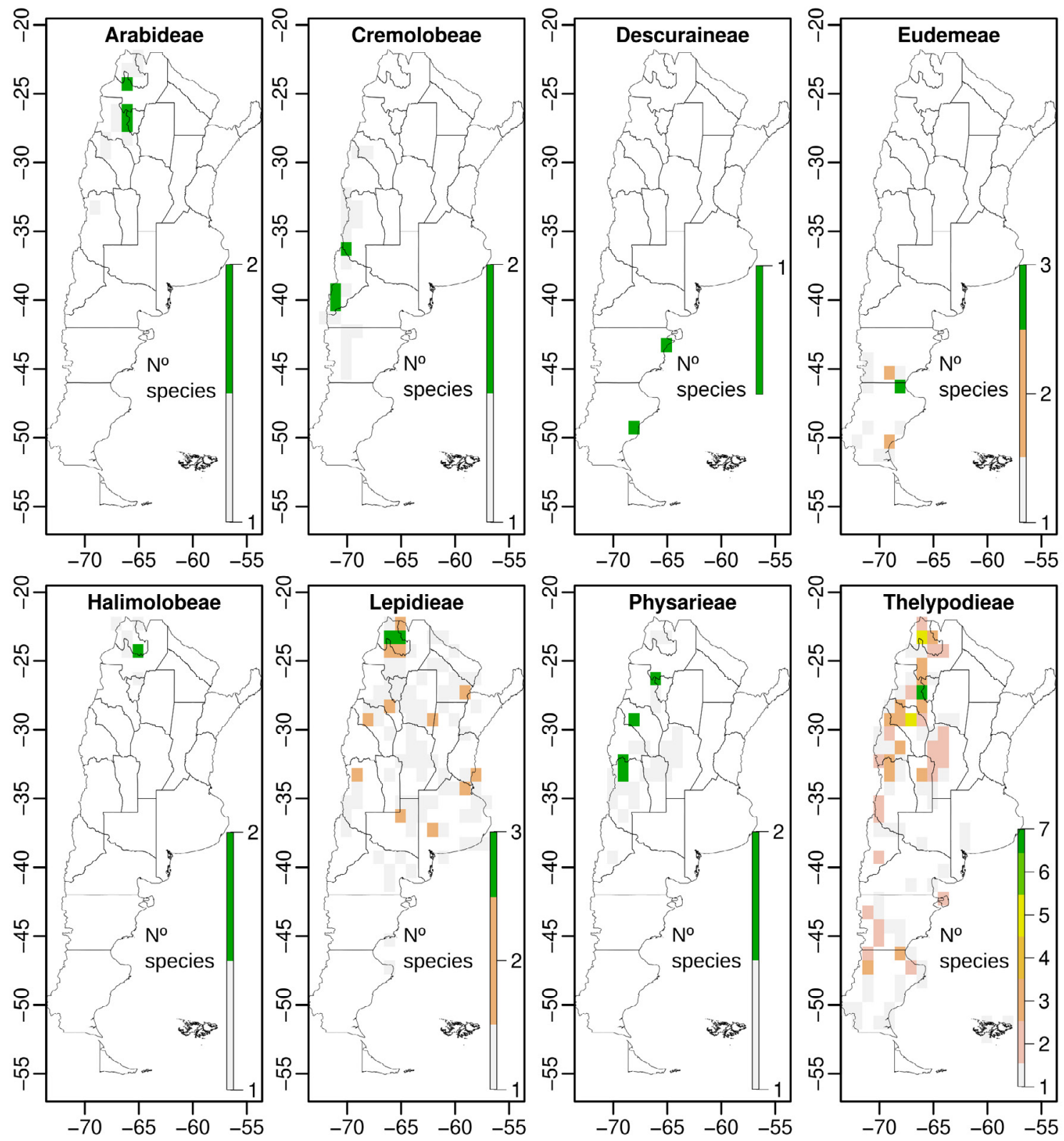

Fig. S3. Spatial patterns of endemism (number of endemic species) for tribes of Brassicaceae family in Argentina based on a spatial grid of 1 arc-degrees. Color version at http://www.ojs.darwin.edu.ar/index.php/darwiniana/article/ view/922/1199 
D. L. SALARIATO \& F. O. ZULOAGA. Diversity and conservation status of crucifers
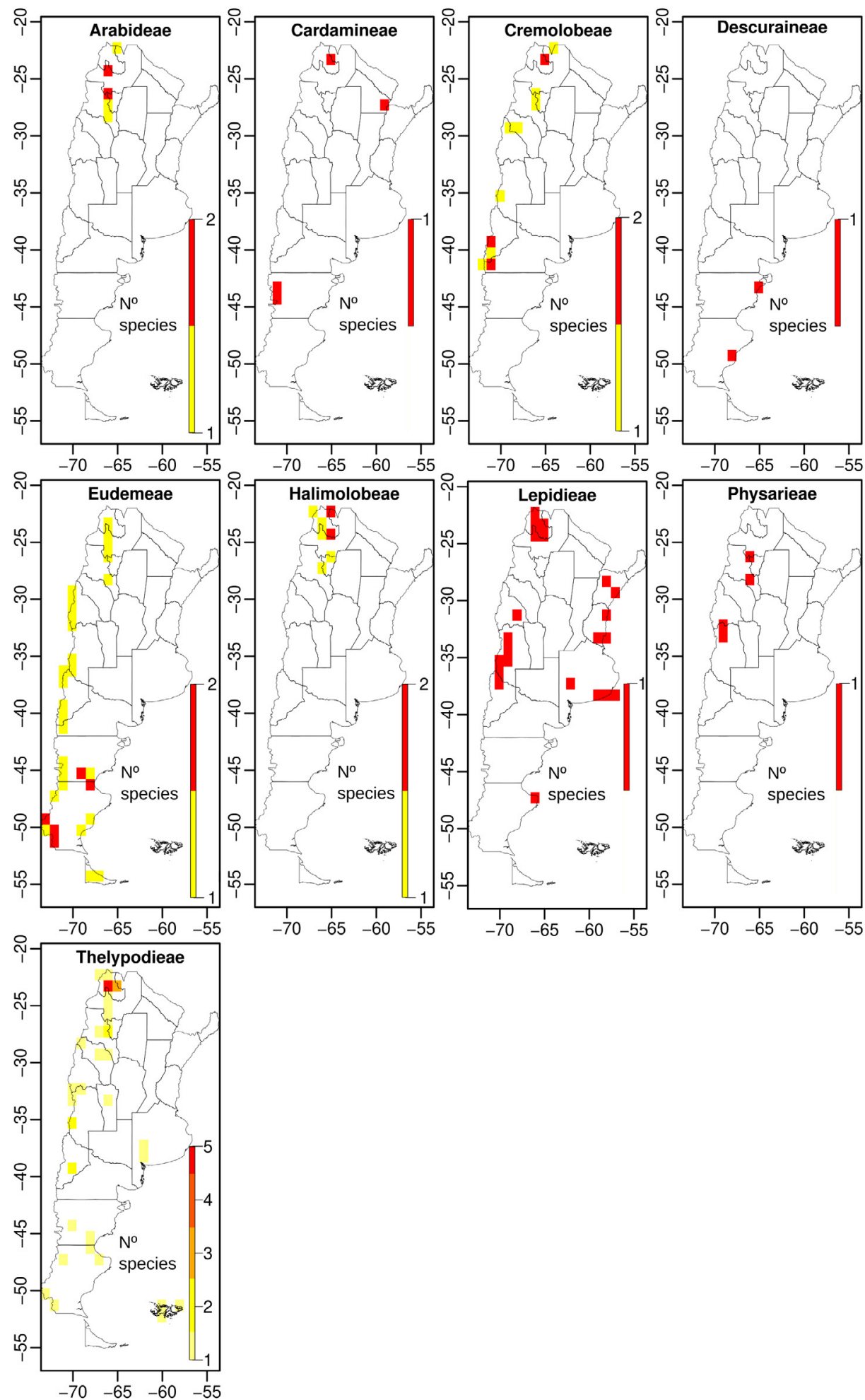

Fig. S4. Spatial patterns of threat (number of threatened species) for tribes of Brassicaceae family in Argentina based on a spatial grid of 1 arc-degrees. Color version at http://www.ojs.darwin.edu.ar/index.php/darwiniana/article/view/922/1199 

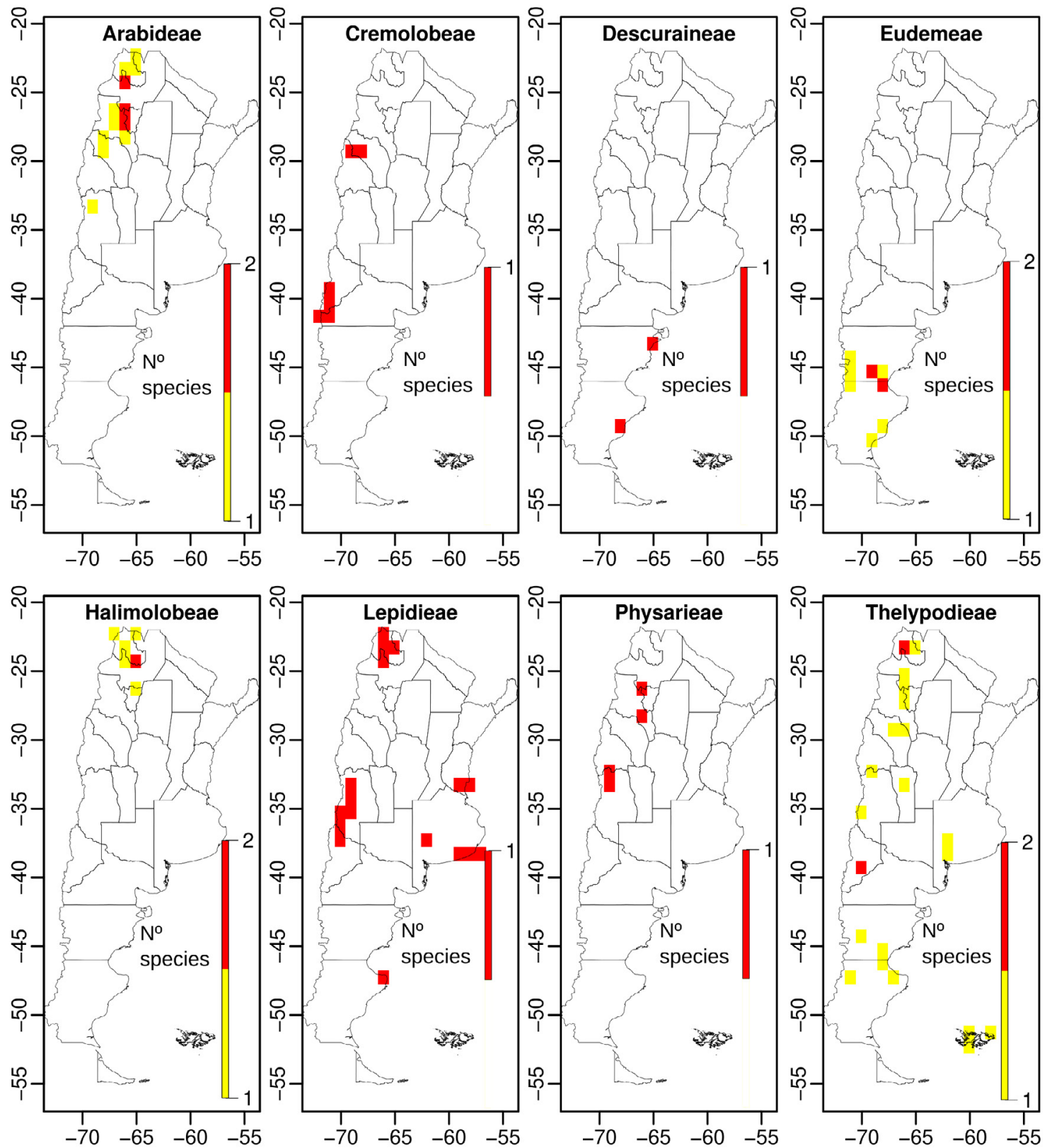

Fig. S5. Spatial patterns of threat for endemics (number of threatened endemic species) for tribes of Brassicaceae family in Argentina based on a spatial grid of 1 arc-degrees. Color version at http://www.ojs.darwin.edu.ar/index.php/ darwiniana/article/view/922/1199 
D. L. SALARIATO \& F. O. ZULOAGA. Diversity and conservation status of crucifers

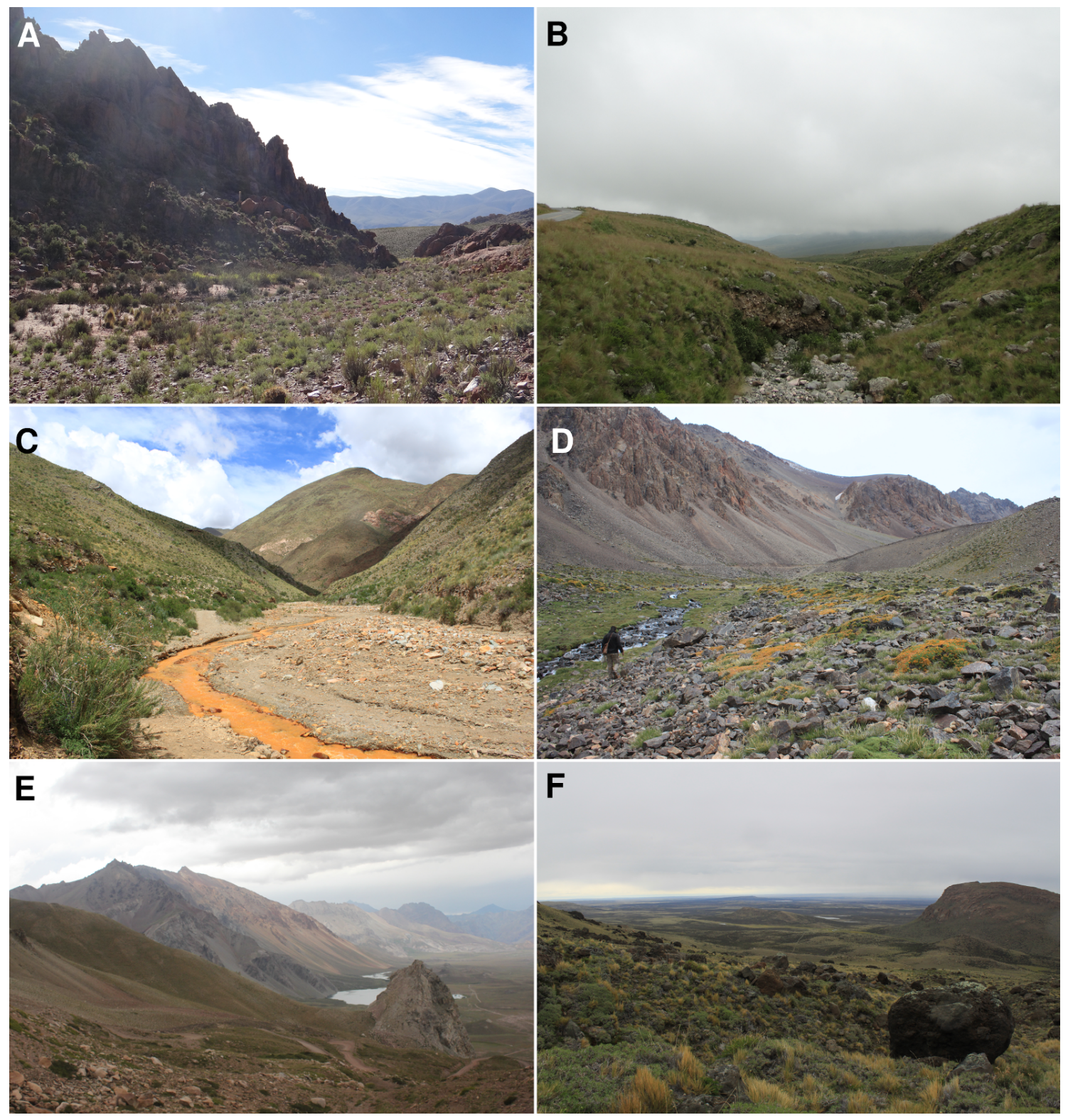

Fig. S6. Some localities associated to cells with high crucifer richness. A, "Espinazo del Diablo" in "Sierra del Aguilar", Jujuy Province (c1) $\left(\sim 23.21^{\circ} \mathrm{S} 65.60^{\circ} \mathrm{W}\right)$. B, "Abra del Infiernillo" in "Tafi", Tucumán province (c2) $\left(\sim 26.73^{\circ} \mathrm{S}\right.$ $\left.65.76^{\circ} \mathrm{W}\right)$. C, Canyon of the "Ocre" river, "Sierra de Famatina", La Rioja province (c5) ( $\left.28.91^{\circ} \mathrm{S} 67.68^{\circ} \mathrm{W}\right)$. D, Near "Portillo Argentino", Mendoza province (c6) $\left(\sim 33.61^{\circ} \mathrm{S} 69.56^{\circ} \mathrm{W}\right)$. E, "Valle Hermoso", Mendoza province (c7) $\left(\sim 35.12^{\circ} \mathrm{S} 70.18^{\circ} \mathrm{W}\right)$. F, “Cerro de la Virgen”, Santa Cruz Province (c9) $\left(\sim 50.78^{\circ} \mathrm{S} 72.23^{\circ} \mathrm{W}\right)$. Color version at http:// www.ojs.darwin.edu.ar/index.php/darwiniana/article/view/922/1199 


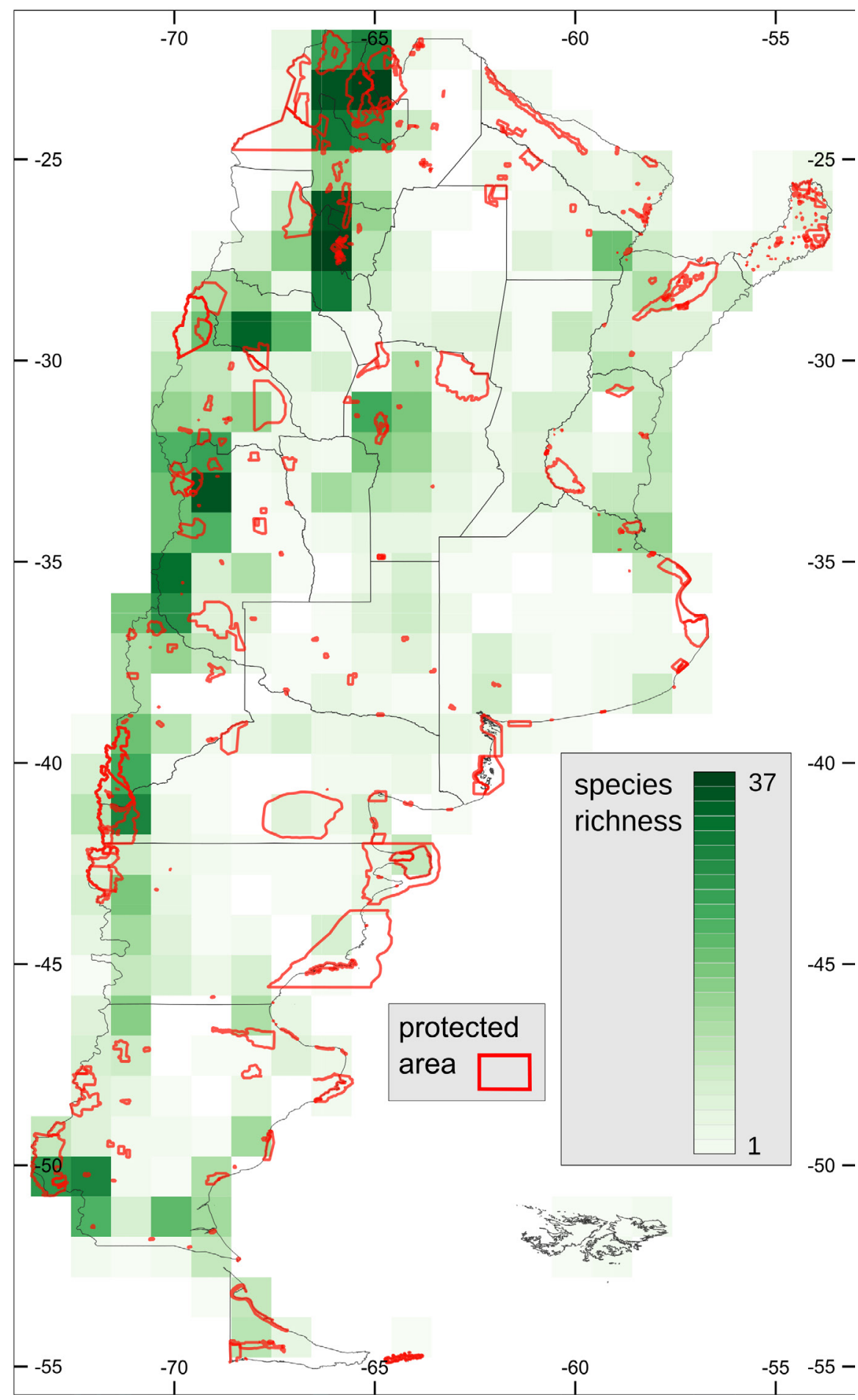

Fig. S7. Species richness for Brassicaceae family in Argentina based on a spatial grid of 1 arc-degrees and superimposed with the existing protected areas in the country. Green gradient represents the number of species within each cell, red lines represent protected areas in Argentina (national, provincial, and biosphere reserves). Color version at http://www. ojs.darwin.edu.ar/index.php/darwiniana/article/view/922/1199 


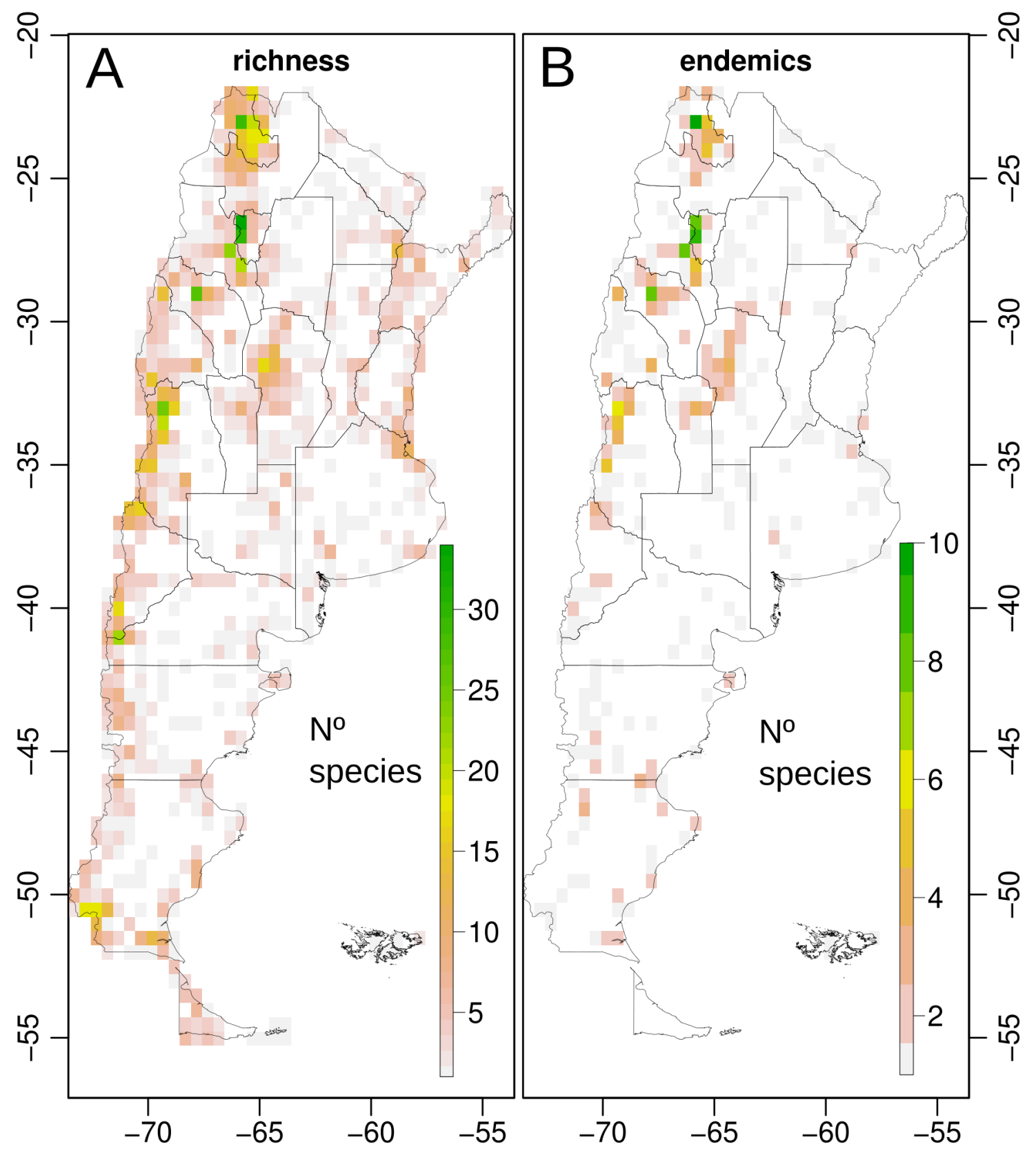

Fig. S8. Spatial patterns of biodiversity for Brassicaceae family in Argentina based on a spatial grid of 0.5 arc-degrees. A, Number of species (richness). B, Number of endemics (endemicity). Color version at http://www.ojs.darwin.edu.ar/ index.php/darwiniana/article/view/922/1199 


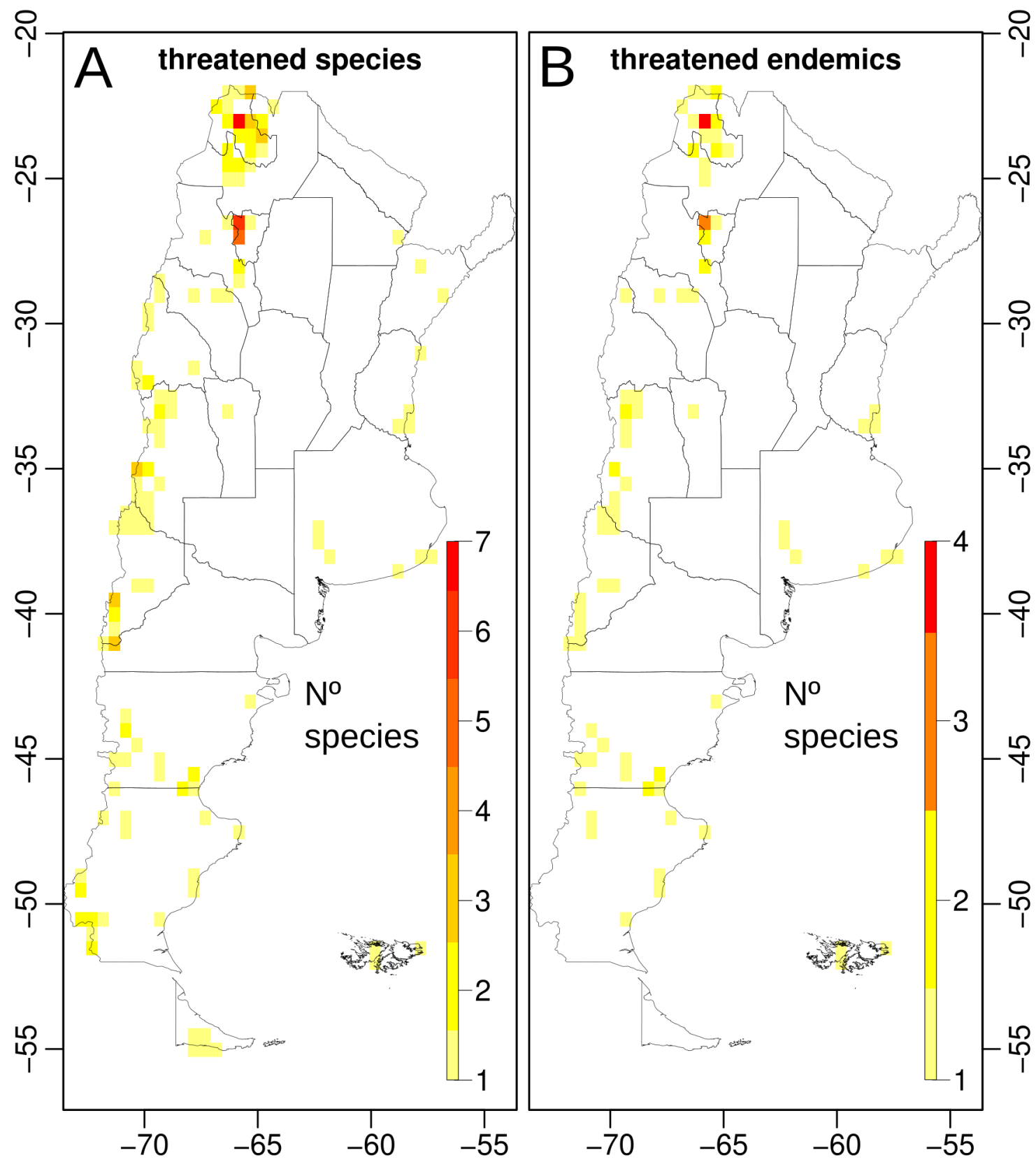

Fig. S9. Spatial patterns of threat for Brassicaceae family in Argentina based on a spatial grid of 0.5 arc degrees. A, Number of threatened species. B, Number of threatened endemic species. Color version at http://www.ojs. darwin.edu.ar/index.php/darwiniana/article/view/922/1199 